\title{
SYSTEMS OF SINGULAR INTEGRAL OPERATORS ON SPHERES
}

\author{
BY
}

DANIEL A. LEVINE

I. This paper has two purposes: to develop a theory of special functions for SO $(n) / S O(n-2)$; and to apply it to the study of systems of singular integral operators on $S^{n-1}$ having specified transformation properties under the action of rotations. The results on special functions for $\mathrm{SO}(n) / \mathrm{SO}(n-2)$ enable us to classify all "irreducible" systems and to decompose an arbitrary singular operator as a sum of operators equivalent (modulo the smoothing operators) to an element of one of the irreducible systems.

To motivate the study of systems of operators, and to illustrate the point of view adopted, we ask the following question about operators on $L^{p}\left(E_{n}\right)$. Which bounded operators on each $L^{p}\left(E_{n}\right), 1<p<\infty$, or merely on $L^{2}\left(E_{n}\right)$, enjoy the invariance properties given below $\left({ }^{1}\right)$ ?

(1) $\tau_{h} T=T \tau_{h}$ for each $h \in E_{n}$, where $\left(\tau_{h} f\right)(x)=f(x-h)$;

(2) $\delta_{\lambda} T=T \delta_{\lambda}$ for each real number $\lambda>0$, where $\left(\delta_{\lambda} f\right)(x)=f\left(\lambda^{-1} x\right)$;

(3) $L_{a} T=T L_{a}$ for each rotation $a$, where $\left(L_{a} f\right)(x)=f\left(a^{-1} x\right)$.

Conditions (1) and (2) show that $(T f)^{\wedge}(\xi)=m(\xi) \hat{f}(\xi)$, where $m(\xi)$ is a homogeneous function of degree 0 , and $\hat{f}$ is the Fourier transform of $f$. If condition (3) is satisfied then $m$ must be constant, and $T$ a scalar multiple of the identity. Therefore, to obtain a nontrivial answer we must relax at least one of the three conditions. To discuss translation-invariant singular operators we keep (1) and (2). (Keeping (1) and (3) leads to the theory of "variable-kernel" operators, to which we shall return shortly.) We relax (3) by asking instead if there is a (complex) vector space $V$ of operators such that the action $T \stackrel{a}{\rightarrow} L_{a} T L_{a}^{-1}$ yields an (irreducible) representation $a \rightarrow R_{a}$ of the rotation group SO (n) on $V$. In other words, as an equality in $V$,

$$
L_{a} T L_{a}^{-1}=R_{a} T
$$

for each $T \in V$ and $a \in \mathrm{SO}(n)$. If $a \rightarrow R_{a}$ is the trivial representation given by $R_{a}=I$ for each $a$, we recover condition (3). If $a \rightarrow R_{a}^{1}=a$ is the standard representation of $\mathrm{SO}(n)$, the system spanned by the Riesz operators $R_{1}, \ldots, R_{n}$ defined by $\left(R_{j} f\right)^{\wedge}(\xi)=\xi_{j}|\xi|^{-1} f(\xi)$ satisfies (1), (2) and (3'). Moreover, as a consideration of $(T f)^{\wedge}(\xi)$ shows, any system satisfying (1), (2) and $\left(3^{\prime}\right)$ for the standard representation must coincide with the span of the Riesz operators. As a generalization of the

Received by the editors February 18, 1969.

(1) The question and some of the discussion that follows is taken from [23, Chapter III] and is given here to help develop the setting for considering systems of singular operators. 
two examples just given, we consider the irreducible representation $a \rightarrow R_{a}^{s}$ of SO $(n)$ on the spherical harmonics of degree $s$. The system spanned by all $s$-fold iterates of the Riesz operators has the required transformation properties, and is the only one that does. In a certain sense these are enough examples for $E_{n}$. In [5] it is shown that any translation-invariant singular operator $T$ can be written as $T=\sum_{s=0}^{\infty} T_{s}$, with $T_{s}$ lying in the system described above for $a \rightarrow R_{a}^{s}$. Moreover, any "variable-kernel" operator $T$ can be written as $T=\sum_{s=0}^{\infty} A_{s} T_{s}$, where $T_{s}$ is as before, and $A_{s}$ is the operator given by multiplication by the bounded smooth function $a_{s}(x)$. In both cases the series converges in $L^{p}$-operator norm.

We now pose a similar question for singular operators on $S^{n-1}$. We ask which irreducible representations $R$ of SO $(n)$ will support a system $V$ of operators $T$ satisfying

$$
L_{a} T L_{a}^{-1}=R_{a} T
$$

for each $a \in \mathrm{SO}(n)$. Also, for the representations that do, we ask how many "different" ones are possible. Here the answer is different than the one sketched above for $E_{n}$. To understand why, we must consider the action of SO (n) on the symbol functions. For $E_{n}$ these are functions on the sphere $S^{n-1}$ (or its Cartesian product with $E_{n}$ ), while for $S^{n-1}$ these are functions on $C S\left(S^{n-1}\right)$, a compact manifold that may be identified with SO $(n) / \mathrm{SO}(n-2)$. (See (1.2) and the remarks on identification following (1.5).)

In §II we investigate in detail the Fourier analysis (Peter-Weyl expansion) of a function in $L^{2}(\mathrm{SO}(n) / \mathrm{SO}(n-2))$. The results obtained are in close analogy to the classical decomposition

$$
L^{2}\left(S^{n-1}\right)=\sum_{s=0}^{\infty} H_{s}
$$

where $H_{s}$ denotes the space of restrictions to $S^{n-1}$ of homogeneous harmonic polynomials of degree $s$ in $x_{1}, \ldots, x_{n}$. We show that in the Peter-Weyl decomposition

$$
L^{2}(\mathrm{SO}(n) / \mathrm{SO}(n-2))=\sum_{m} \oplus \mathscr{H}_{m}
$$

the space $\mathscr{H}_{m}$ can be realized as the restriction to the manifold

$$
C S\left(S^{n-1}\right)=\left\{(x, \xi) \in E_{n} \times\left. E_{n}\right|_{\xi_{1}^{2}+\cdots+\xi_{n}^{2}=1} ^{x_{2}^{2}+\cdots+x_{2}^{2}=1} x_{1} \xi_{1}+\cdots+x_{n} \xi_{n}=0\right\}
$$

of a space $Q_{m}$ of polynomials in $x_{1}, \ldots, x_{n}, \xi_{1}, \ldots, \xi_{n}$ that have the desired transformation behavior (Theorem 2). It is these spaces that are used in $\S \mathrm{IV}$ to construct operators having specified transformation laws analogous to (1.1). We show that the polynomials in $Q_{m}$ are harmonic in a sense related to the action of $\mathrm{SO}(n)$ on $E_{n} \times E_{n}$ given by (2.3), and that $\sum_{m} Q_{m}$ is in fact the space of all harmonic polynomials (Theorem 3). The chief difference between (1.2) and (1.3) is that while $H_{s}$ 
is irreducible under the action of SO $(n), \mathscr{H}_{m}$ is in general a sum of more than one irreducible subspace.

Estimates for the operator $\Lambda^{-1}$, which plays a role analogous to the $\Lambda^{-1}$ introduced by Calderón and Zygmund, are proved in §III. The description of $\Lambda^{-1}$ in local coordinates is carried out there as well. In $\S I V$ we give definitions and pose the problem on the existence of systems in a more precise form. We construct canonical systems that are analogous to the examples presented above for $E_{n}$, and prove a decomposition theorem of an arbitrary singular integral operator on $L^{p}\left(S^{n-1}\right)$ (Theorem 5). This decomposition theorem allows us to classify all possible systems of singular operators transforming according to an irreducible representation of SO $(n)$ (Theorem 6).

The contents of this paper form most of the author's doctoral dissertation, written for Princeton University under the direction of Professor E. M. Stein. It is with great pleasure that the debt owed him is here recorded.

Notation. Most notation will be explained as it is introduced. In general, $E_{k}$ will denote $k$-dimensional Euclidean space and $x=\left(x_{1}, \ldots, x_{k}\right)$ a point in this space. The norm of $x$ will be denoted by $|x|$. The multi-index convention for differentiation in $E_{k}$ will often be used. Thus if $\alpha=\left(\alpha_{1}, \ldots, \alpha_{k}\right)$

$$
\frac{\partial^{|\alpha|} f}{\partial x^{\alpha}}=\left(\frac{\partial}{\partial x}\right)^{\alpha} f=\frac{\partial^{\alpha_{1}+\cdots+\alpha_{k}}}{\partial x_{1}^{\alpha}{ }_{1} \cdots \partial x_{k}^{\alpha} k} f
$$

The Fourier transform of a function $f$ in $L^{1}\left(E_{k}\right)$ or $L^{2}\left(E_{k}\right)$ will be denoted $\hat{f}$ and defined by

$$
\hat{f}(\xi)=\int_{E_{k}} f(x) e^{i x \xi} d x
$$

By SO (n) we denote group of rotations in $E_{n}$ that preserve orientation.

IDENTIFICATIONS. The group $S O(n)$ acts transitively on $S^{n-1}=\left\{x \in E_{n}|| x \mid=1\right\}$ by the action $x \rightarrow a x$, where $a=\left(a_{i j}\right)$ is regarded as an $n \times n$ matrix and $x$ as a column vector. The fixing group of the "north pole" $N=(0, \ldots, 0,1)$ is a copy of $\mathrm{SO}(n-1)$, and so we have the identification $S^{n-1} \cong \mathrm{SO}(n) / \mathrm{SO}(n-1)$. The symbol $\sigma(T)$ of a singular integral operator $T$ on $L^{p}\left(S^{n-1}\right)$ is a function on the cotangent bundle $T^{*}\left(S^{n-1}\right)$, which may be described as

$$
T^{*}\left(S^{n-1}\right)=\left\{(x, \xi) \in E_{n} \times E_{n}|| x \mid=1, x \xi=0\right\},
$$

where $x \xi=x_{1} \xi_{1}+\cdots+x_{n} \xi_{n}$. For each fixed $x$ we may introduce a metric in the fibre over $x$,

$$
T_{x}^{*}\left(S^{n-1}\right)=\left\{(x, \xi) \in\{x\} \times E_{n} \mid x \xi=0\right\}
$$

by use of the norm

$$
|\xi|=\left(\xi_{1}^{2}+\cdots+\xi_{n}^{2}\right)^{1 / 2}
$$


where $\xi$ stands for $(x, \xi) \in T_{x}^{*}$. This metric depends differentiably on $x$. Since it is homogeneous and $\sigma(T)$ is homogeneous of degree 0 on each fibre of $T^{*}\left(S^{n-1}\right)$, $\sigma(T)$ is actually determined by its value on the cosphere bundle of $S^{n-1}$ (see [21]), which may thus be written as

$$
C S\left(S^{n-1}\right)=\left\{(x, \xi) \in E_{n} \times\left. E_{n}\right|_{|\xi|=1} ^{|x|=1} x \xi=0\right\} .
$$

The group SO (n) acts transitively on $C S\left(S^{n-1}\right)$, and the fixing group of the point $\left(N, v_{0}\right)$ is $\left\{\left(a_{i j}\right) \in \mathrm{SO}(n) \mid a_{n n}=a_{n-1, n-1}=1\right\}$, where $v_{0}=(0, \ldots, 0,1,0)$. Thus $C S\left(S^{n-1}\right)$ can be diffeomorphically identified with SO $(n) / S O(n-2)$. When we use the symbol $C S\left(S^{n-1}\right)$ we shall always mean the manifold given by (1.7), and when we write $C S\left(S^{n-1}\right) \cong S O(n) / S O(n-2)$ we shall regard SO $(n-2)$ as the fixing group of the point $\left(N, v_{0}\right)$ defined above.

The action of SO $(n)$ on SO $(n) / \mathrm{SO}(n-2)$ induced by $g \cdot \mathrm{SO}(n-2) \stackrel{a}{\rightarrow}(a g)$ -SO $(n-2)$, when transferred to $C S\left(S^{n-1}\right)$ by the identification described above, becomes

$$
(x, \xi) \stackrel{a}{\longrightarrow}(a x, a \xi) .
$$

If we compute the action on $T^{*}\left(S^{n-1}\right)$ induced by the action $x \stackrel{a}{\rightarrow} a x$ of $\mathrm{SO}(n)$ on $S^{n-1}$, the result is also (1.8). We shall rely heavily on this last remark in several places. It may be restated as follows: if $l_{a}(x)=a x$, then

$$
l_{a}^{*}(x, \xi)=(a x, a \xi),
$$

where $l_{a}^{*}$ is the map: $T_{a x}^{*}\left(S^{n-1}\right) \rightarrow T_{x}^{*}\left(S^{n-1}\right)$ induced by $l_{a}$.

Action of SO ( $n)$ on operators and symbols. If $T$ is a singular integral operator in a suitable class, then so is $L_{a} T L_{a}^{-1}$. To be specific, whenever we say $T$ is a singular operator we shall mean that $T$ lies in the class $C_{\infty}^{\infty}\left(S^{n-1}\right)$ defined by Seeley in [21]. The requirements thus imposed on $T$ may be summarized by saying that the transfer of $T$ to any coordinate neighborhood $U$ yields an operator of the form $\left(T_{U} f\right)(x)=a(x) f(x)+\int_{E_{n-1}} k(x, x-y) f(y) d y+(R f)(x), x \in U$. Here $k(x, z)$ is to be a Calderón-Zygmund kernel that is $C^{\infty}$ for $z \neq 0, a(x)$ a bounded smooth function, and $R$ a "smoothing" operator.

The symbols $\sigma(T)$ and $\sigma\left(L_{a} T L_{a}^{-1}\right)$ are related by a transformation law:

$$
\sigma(T)(x, \xi)=\sigma\left(L_{a} T L_{a}^{-1}\right)(a x, a \xi) .
$$

This transformation law will be derived in §IV in a slightly different context (Proposition 10).

Representations of $\mathrm{SO}(n)$ and integration against characters. The irreducible representations of $\mathrm{SO}(n)$ are in one-to-one correspondence with dominant weight vectors $m=\left(m_{1}, \ldots, m_{k}\right)$, where $k=[n / 2]$. We require that the entries be nonnegative integers (we shall not consider double-valued representations) satisfying $m_{1} \geqq m_{2} \geqq$ $\cdots \geqq m_{k} \geqq 0$ for $n$ odd and $m_{1} \geqq m_{2} \geqq \cdots \geqq\left|m_{k}\right|$ for $n$ even. We shall denote the 
representation corresponding to the dominant weight vector $m$ by $R^{m}$. See [24] for a brief discussion of this correspondence, or [1] or [25] for a complete discussion.

We shall have occasion to use the following easily verified fact about the characters of irreducible representations of a compact group. Let $R$ be an irreducible representation of the compact group $G$ that is a subrepresentation of a representation $L$ of $G$ on a Hilbert space $\mathscr{H}$. Then the projection $\pi_{R}$ from $\mathscr{H}$ onto the closed subspace $\mathscr{H}_{R}$ of $\mathscr{H}$ consisting of all the copies of irreducible spaces on which the action of $L$ is equivalent to that of $R$ is given by

$$
\pi_{R} v=d_{R} \int_{G}\left(L_{a} v\right) \bar{\chi}_{R}(a) d a,
$$

where $d_{R}$ is the degree of $R$ and $\chi_{R}$ is its character. Here as throughout, the Haar measure $d a$ on $\mathrm{SO}(n)$ will be assumed to be normalized so that $\int_{\mathrm{So}(n)} d a=1$.

II. We begin by recapitulating in Theorem 1 the results of the Peter-Weyl theorem as they apply to SO $(n) / S O(n-2) \cong C S\left(S^{n-1}\right)$. Before defining the spaces $P_{r, s}^{m}$ and $Q_{m}$ that will be used to set up a correspondence between symbol functions and operators (in $\S I V$ ), we define the notion of an SO (n)-harmonic polynomial. After showing that the restriction map to $C S\left(S^{n-1}\right)$ has kernel 0 on $Q_{m}$ (Theorem 2 ), we show that the polynomials in $Q_{m}$ are $S O(n)$-harmonic (Theorem 3 ). The Corollary of Theorem 3 may be interpreted as explaining the fact that the restriction map has kernel 0 on $Q_{m}$.

Let $L$ denote the left regular representation of SO $(n), n \geqq 3$, on

$$
L^{2}(\mathrm{SO}(n) / \mathrm{SO}(n-2))
$$

defined by $\left(L_{a} f\right)(x)=f\left(a^{-1} x\right)$ for $f \in L^{2}(\mathrm{SO}(n))$.

THEOREM 1.

$$
L^{2}(\mathrm{SO}(n) / \mathrm{SO}(n-2))=\sum_{m} \oplus \mathscr{H}_{m},
$$

where each $\mathscr{H}_{m}$ is an invariant subspace such that the restriction $L \mid \mathscr{H}_{m}=\sum_{i=1}^{s_{m}} \oplus R^{m, i}$. In the first sum the summation is over all dominant weights $m$ of the form $\left(m_{1}, m_{2}, 0\right.$, $\ldots, 0)$. In the second sum $s_{m}=m_{1}-\left|m_{2}\right|+1$ for $n \geqq 4, s_{m}=2 m+1$ for $n=3$, and $R^{m, i}$ are copies of the irreducible representation $R^{m}$ corresponding to the dominant weight $m$.

Proof. For $n=3$ the statement is part of the Peter-Weyl theorem applied to the left regular representation of SO (3) on $L^{2}(\mathrm{SO}(3))$. For $n \geqq 4$ we apply the PeterWeyl theorem to conclude that $L=\sum_{m} \oplus c_{m} R^{m}$. The coefficients $c_{m}$ can be computed as follows. Let $V^{m}$ be a representation space for $R^{m}$. By applying the branching theorem twice, (see [1, pp. 251-253]), first on restricting $R^{m}$ to SO $(n-1)$, and then on restricting each irreducible subrepresentation of $\left.R^{m}\right|_{\text {so }(n-1)}$ to $\mathrm{SO}(n-2)$, we see that an $m_{1}-\left|m_{2}\right|+1$-dimensional subspace of $V^{m}$ is left invariant by $\left.R^{m}\right|_{\text {so }(n-2)}$. The conclusion then follows from the Frobenius reciprocity theorem. 
We pass to the notion of a $G$-harmonic polynomial. The terminology and notation is from [8]. Let $V$ be a finite dimensional real vector space and let $S\left(V^{*}\right)=$ the polynomial functions on $V$. Suppose $G$ acts on $V$ by the action $v \stackrel{g}{\rightarrow} g \cdot v$. Then it acts on $V^{*}$ by $\left(g \cdot v^{*}\right)(v)=v^{*}\left(g^{-1} \cdot v\right)$, and this action can be extended to $S\left(V^{*}\right)$.

Now each element $X \in V$ gives rise (by parallel translation) to a vector field on $V$, which we regard as a differential operator $\partial(X)$ on $V$. This mapping $X \rightarrow \partial(X)$ extends to an isomorphism of the symmetric algebra $S(V)$ over $V$ onto the algebra of all differential operators on $V$ with constant complex coefficients. We let $I(V)$ denote the image of the elements of $S(V)$ invariant under the action of $\mathrm{SO}(n)$, and $I_{+}(V)$ denote the image of those elements of $I(V)$ with zero constant term.

Definition. An element $p \in S\left(V^{*}\right)$ is a $G$-harmonic polynomial if it is annihilated by all invariant differential operators (of positive degree):

$$
\partial(J) p=0 \text { for } J \in I_{+}(V) .
$$

If we take $V$ to be a real $n$-dimensional vector space with basis $\left\{\partial / \partial x_{1}, \ldots, \partial / \partial x_{n}\right\}$, choose the dual basis $\left\{x_{1}, \ldots, x_{n}\right\}$ for $V^{*}$ and let $\mathrm{SO}(n)$ act on $V^{*}$ by $x \stackrel{a}{\rightarrow} a x$, where $x$ is regarded as a column vector, then we recover the classical definition of a harmonic polynomial in $x_{1}, \ldots, x_{n}$. For our purposes we shall take $G=\operatorname{SO}(n)$ and let $V$ be a real vector space of dimension $2 n$ with basis $\left\{\partial / \partial x_{1}, \ldots, \partial / \partial x_{n}\right.$, $\left.\partial / \partial \xi_{1}, \ldots, \partial / \partial \xi_{n}\right\}$. We choose the dual basis $\left\{x_{1}, \ldots, x_{n}, \xi_{1}, \ldots, \xi_{n}\right\}$ for $V^{*}$ and let SO $(n)$ act on $V^{*}$ by the action

$$
(x, \xi) \stackrel{a}{\longrightarrow}(a x, a \xi) .
$$

Here $x$ and $\xi$ are to be regarded as column vectors. When we speak of a "harmonic" polynomial in $x_{1}, \ldots, x_{n}, \xi_{1}, \ldots, \xi_{n}$ it will always refer to the action (2.3) of SO (n).

Eventually (Theorem 3 ) we shall show that the spaces $P_{r, s}^{m}$ and $Q_{m}$ we are about to define are spaces of SO $(n)$-harmonic polynomials in the sense of the above definition. Before beginning the proofs of Theorems 2 and 3, we write down all the necessary definitions and give the statement of these theorems.

Let $n \geqq 3$, and let $m=\left(m_{1}, m_{2}, 0, \ldots, 0\right)$ be a dominant weight of the form discussed in Theorem 1. Let $r$ and $s$ be any two nonnegative integers such that

$$
r+s=m_{1}+\left|m_{2}\right|, \quad\left|m_{2}\right| \leqq r \leqq m_{1} .
$$

Let us realize $R^{(r, 0, \ldots, 0)}$ on $H_{r}(x)=\{$ (solid) homogeneous harmonic polynomials in variables $x_{1}, \ldots, x_{n}$ of degree $r$, complex coefficients $\}$, and similarly, let us realize $R^{(s, 0, \ldots, 0)}$ on $H_{s}(\xi)=\{$ (solid) homogeneous harmonic polynomials in variables $\xi_{1}, \ldots, \xi_{n}$ of degree $s$, complex coefficients $\}$. Then $H_{r}(x) \otimes H_{s}(\xi)$ can be regarded as a vector space of polynomials $p(x, \xi)$ such that $p(t x, u \xi)=t^{n} u^{s} p(x, \xi)$. The tensor product $R^{(r, 0, \ldots, 0)} \otimes R^{(s, 0, \ldots, 0)}$ acts on $H_{r}(x) \otimes H_{s}(\xi)$, but not irreducibly.

LEMMA 1. Let $r$ and s be nonnegative integers with $s \leqq r$. Let $R^{\lambda}$ denote the irreducible representation of $\mathrm{SO}(n)$ corresponding to the dominant weight $(\lambda, 0, \ldots, 0)$ and let 
$R^{\lambda_{1}, \lambda_{2}}$ denote the one corresponding to the dominant weight $\left(\lambda_{1}, \lambda_{2}, 0, \ldots, 0\right)$. Then for $n \geqq 4$ the tensor product breaks down as follows into irreducible representations, each with multiplicity 1 :

$$
\begin{aligned}
R^{r} \otimes R^{s}= & R^{r+s} \oplus R^{r+s-2} \oplus \cdots \oplus R^{r-s} \\
& \oplus R^{r+s-1,1} \oplus R^{r+s-3,1} \oplus \cdots \\
& \oplus \cdots \\
& \oplus R^{r, s} .
\end{aligned}
$$

This decomposition is given in [15, p. 276], for the group $O(n)$. All the representations on the right side of $(2.5)$ remain irreducible on restriction to SO $(n)$, provided $n \neq 4$. If $n=4$, the irreducible representation $R^{\lambda_{1}, \lambda_{2}}\left(\lambda_{2}>0\right)$ of $O(4)$ decomposes as $R^{\lambda_{1}, \lambda_{2}} \oplus R^{\lambda_{1},-\lambda_{2}}$ on restriction to SO (4). See [1, pp. 96 ff. and p. 262].

We notice that under the restrictions (2.4) on $r$ and $s$ the representation $R^{m}$ occurs exactly once on the right side of (2.5). For such $r$ and $s$ we are in a position to make the following

Definition. The space $P_{r, s}^{m}$ is the irreducible subspace of $H_{r}(x) \otimes H_{s}(\xi)$ (regarded as polynomials in $x_{1}, \ldots, x_{n}, \xi_{1}, \ldots, \xi_{n}$ ) consisting of vectors transforming according to $R^{m}$ under the action (2.3) of SO $(n)$.

Summing over $r$ and $s$ satisfying (2.4) we have the

DEFINITION. For $n \geqq 4$,

$$
Q_{m}=\sum_{r+s=m_{1}+\left|m_{2}\right|:\left|m_{2}\right| \leq r \leq m_{1}} \oplus P_{r, s}^{m}
$$

We note that there are $s_{m}=m_{1}-\left|m_{2}\right|+1$ irreducible summands in (2.6). For $n=3$ we shall adopt a modification that will have $s_{m}=2 m+1$ terms. See (2.14).

Let us recall the expression (1.7) for $C S\left(S^{n-1}\right)$ as well as the remarks made afterwards about the identification of $C S\left(S^{n-1}\right)$ and SO $(n) / S O(n-2)$.

THEOREM 2. Let $n \geqq 4$ and $m=\left(m_{1}, m_{2}, 0, \ldots, 0\right)$ be a dominant weight. The space $Q_{m}$ defined by (2.6) is mapped by the restriction map to $C S\left(S^{n-1}\right)$ into

$$
\mathscr{H}_{m} \subseteq L^{2}(\mathrm{SO}(n) / \mathrm{SO}(n-2)) .
$$

The restriction map commutes with the action of $\mathrm{SO}(n)$ induced on each of these spaces by (2.3). Furthermore, the restriction map has kernel 0 on $Q_{m}$ and carries $Q_{m}$ onto $\mathscr{H}_{m}$.

THEOREM 3. Let $n \geqq 3$. The space $H\left(V^{*}\right)$ of $\mathrm{SO}(n)$-harmonic polynomials in $C\left[x_{1}, \ldots, x_{n}, \xi_{1}, \ldots, \xi_{n}\right]$ under the action (2.4) coincides with the (algebraic) direct sum

$$
\sum_{\left(m_{1}, m_{2}, 0, \ldots, 0\right)} Q_{m}
$$

Here $C\left[x_{1}, \ldots, x_{n}, \xi_{1}, \ldots, \xi_{n}\right]$ denotes the ring of complex coefficient polynomials in $x_{1}, \ldots, x_{n}, \xi_{1}, \ldots, \xi_{n}$. 
COROLlaRY. If $h$ is an SO (n)-harmonic polynomial that vanishes on $\operatorname{CS}\left(S^{n-1}\right)$, then $h$ is the zero polynomial.

REMARK. For the Corollary in a much more general setting see [12].

The proofs of these statements will occupy most of the remainder of this chapter. After the proof of Theorem 2 we shall introduce the modifications necessary in the definition of $Q_{m}$ for the case $n=3$. Theorem 4 will give the results for $n=3$ corresponding to Theorem 2, whose proof we now begin.

If $q \in C\left[x_{1}, \ldots, x_{n}, \xi_{1}, \ldots, \xi_{n}\right]$, we define the polynomial $q_{a}$ by

$$
q_{a}(x, \xi)=q\left(a^{-1} x, a^{-1} \xi\right) .
$$

Let $C S$ stand for $C S\left(S^{n-1}\right)$, and let $\operatorname{Res}_{m}$ denote the restriction map to $C S$ for polynomials in $Q_{m}$. Then if $L$ is the left regular representation discussed in Theorem 1,

$$
\left.q_{a}\right|_{c s}=L_{a}\left(\left.q\right|_{c s}\right)
$$

so that restriction to $C S$ commutes with the action of SO $(n)$, and hence $\operatorname{Res}_{m}$ carries $Q_{m}$ into $\mathscr{H}_{m}$. Since $Q_{m}$ and $\mathscr{H}_{m}$ have the common dimension $s_{m}=m_{1}-\left|m_{2}\right|+1$ as complex vector spaces, Theorem 2 will be proved once we show that the kernel of $\operatorname{Res}_{m}$ is zero.

To do this we shall use the following two propositions, which we prove at the end of this chapter, using the methods of algebraic geometry. Let $S$ denote the ring $C\left[x_{1}, \ldots, x_{n}, \xi_{1}, \ldots, \xi_{n}\right]$.

Proposition 1. Let $n \geqq 2$ and let $q \in S$ be any polynomial that vanishes on $\operatorname{CS}\left(S^{n-1}\right)$. Then there are polynomials $A, B, C$ in $S$ such that

$$
q=A\left(|x|^{2}-1\right)+B\left(|\xi|^{2}-1\right)+C x \xi
$$

Proposition 2. The vector space homomorphism $\phi: S \oplus S \oplus S \rightarrow S$ defined by $\phi(A, B, C)=A|x|^{2}+B|\xi|^{2}+C x \xi$ has as its kernel the ideal in $S$ generated by the three elements

$$
\left(|\xi|^{2},-|x|^{2}, 0\right), \quad\left(x \xi, 0,-|x|^{2}\right), \quad\left(0, x \xi,-|\xi|^{2}\right) .
$$

If $q$ lies in the kernel of $\operatorname{Res}_{m}$, we apply Proposition 1 to it. Writing $A, B$ and $C$ as a sum of homogeneous polynomials:

$$
A=\sum_{j=0}^{s} A_{j}, \quad B=\sum_{j=0}^{s} B_{s}, \quad C=\sum_{j=0}^{s} C_{j}
$$

and substituting the resulting expression (2.8) into (2.7) yields, on equating terms of equal homogeneity:

$$
\begin{aligned}
A_{s}|x|^{2}+B_{s}|\xi|^{2}+C_{s} x \xi & =0 \\
A_{s-2}|x|^{2}+B_{s-2}|\xi|^{2}+C_{s-2} x \xi-A_{s}-B_{s} & =0 \\
\cdot \cdot \cdot & \cdot \\
A_{p-2}|x|^{2}+B_{p-2}|\xi|^{2}+C_{p-2} x \xi-A_{p}-B_{p} & =q \\
\cdot \cdot \cdot & \cdot A_{0}+B_{0}=0
\end{aligned}
$$


where $p$ denotes the total homogeneity $m_{1}+\left|m_{2}\right|$ of $q$. Applying Proposition 2 to the top line of (2.9) yields $A_{s}=\alpha_{s-2}|\xi|^{2}+\beta_{s-2} x \xi, B_{s}=-\alpha_{s-2}|x|^{2}+\gamma_{s-2} x \xi$. (Here we may assume $\alpha_{s-2}, \beta_{s-2}, \gamma_{s-2}$ are homogeneous of degree $s-2$.)

Substituting into the second equation of (2.9) yields

$$
\left(A_{s-2}+\alpha_{s-2}\right)|x|^{2}+\left(B_{s-2}-\alpha_{s-2}\right)|\xi|^{2}+\left(C_{s-2}-\beta_{s-2}-\gamma_{s-2}\right) x \xi=0 .
$$

Applying Proposition 2 to (2.10) yields

$$
\begin{aligned}
& A_{s-2}+\alpha_{s-2}=\alpha_{s-4}|\xi|^{2}+\beta_{s-4} x \xi, \\
& B_{s-2}-\alpha_{s-2}=-\alpha_{s-4}|x|^{2}+\gamma_{s-4} x \xi .
\end{aligned}
$$

We can now substitute these expressions into the third equation of (2.9), and so on, until we arrive at the equation

$$
A_{p}+B_{p}=\alpha_{p-2}|\xi|^{2}-\alpha_{p-2}|x|^{2}+\left(\beta_{p-2}+\gamma_{p-2}\right) x \xi
$$

where $\alpha_{p-2}, \beta_{p-2}$ and $\gamma_{p-2}$ are homogeneous of degree $p-2$. But then, from the equation in (2.9) involving terms of homogeneity $p$,

$$
q=a|x|^{2}+b|\xi|^{2}+c x \xi
$$

where $a, b$ and $c$ are homogeneous of degree $p-2$.

DefinITION. Let $R^{m}$ be the irreducible representation of SO $(n), n \geqq 3$, whose highest weight is $\left(m_{1}, m_{2}, \ldots, m_{k}\right)$. The richness of $R^{m}$ and of $m$ is

$$
|m|=\left|m_{1}\right|+\cdots+\left|m_{k}\right| \text {. }
$$

It is easy to show that if $|m|=r$, then $R^{m}$ occurs as a subrepresentation of $\otimes^{r} s t_{n}$ acting on $\otimes^{r} E_{n}$, where $s t_{n}$ denotes the standard representation $a \rightarrow a$ of SO (n) acting as rotations on $E_{n}$. See [24]. We shall use a converse of this fact:

LEMMA 2. Any subrepresentation of $\otimes^{r} s_{n}$ has richness at most $r$.

The proof is a simple argument by induction, using the fact that if $R$ is an irreducible representation of richness $t$, then $R \otimes s t_{n}$ breaks up into irreducible representations whose richness is at most $t+1$. See the discussion of Cartan composition in [24].

It follows from Lemma 2 and the use of the identification mentioned just before Lemma 1 that in the decomposition into irreducible subspaces of

$$
S_{t}=\{p \in S \mid \text { degree } p \leqq t\}
$$

under the action $q \stackrel{a}{\rightarrow} q_{a}$ of SO (n) none of the irreducible representations that occur has richness exceeding $t$.

Now it follows from the projection formula (1.10) that

$$
q(x, \xi)=d_{m} \int_{\text {so }(n)} q_{g}(x, \xi) \bar{\chi}_{m}(g) d g .
$$


Substituting the expression (2.11) on the right side of (2.12) we obtain

$$
\begin{aligned}
q(x, \xi)= & d_{m}|x|^{2} \int_{\mathrm{SO}(n)} a_{g}(x, \xi) \bar{\chi}_{m}(g) d g+d_{m}|\xi|^{2} \int_{\mathrm{SO}(n)} b_{g}(x, \xi) \bar{\chi}_{m}(g) d g \\
& +d_{m}|x|^{2} \int_{\mathrm{SO}(n)} c_{g}(x, \xi) \bar{\chi}_{m}(g) d g .
\end{aligned}
$$

But each of the integrals on the right must be zero, since the richness of $R^{m}$ is $p=$ degree $q$, while $a, b$ and $c$ lie in $S_{p-2}$. This completes the proof of Theorem 2 .

The case $n=3$ is atypical in that the multiplicity of $H_{r}$ in $\mathscr{H}_{r}$ is $2 r+1$. Let us consider first the example of $R^{1}$, which occurs three times in $L^{2}$ (SO (3)). We may realize it in two "different" ways on $H_{1}(x)$ and $H_{1}(\xi)$ respectively. The proof of Theorem 5.2 applies so far. To find the "other" realization we use the fact that for $n=3$ the adjoint representation "degenerates" into the standard representation $R^{1}$. Guided by the construction performed for the adjoint representation in the case $n \geqq 5$ (for $n=4$ the adjoint representation is not irreducible), we look at $H_{1}(x) \otimes H_{1}(\xi)$ and select the three dimensional space spanned by the polynomials $x_{1} \xi_{2}-x_{2} \xi_{1}, x_{1} \xi_{3}-x_{3} \xi_{1}, x_{2} \xi_{3}-x_{3} \xi_{2}$. Here we resort to using polynomials of degree 2 to get all the representations we need.

The Clebsch-Gordan formula for SO (3) is

$$
R^{r} \otimes R^{s}=R^{r+s} \oplus R^{r+s-1} \oplus \cdots \oplus R^{r-s}
$$

for $0 \leqq s \leqq r$ (see (2.5)). To get all the spaces we need for $R^{m}$ we allow $r$ and $s$ to be nonnegative integers such that $0 \leqq s \leqq r \leqq m$ and $r+s$ is either $m$ or $m+1$. This determines $2 m+1$ products that contain $R^{m}$ acting on a space $P_{r, s}^{m} \subseteq H_{r}(x) \otimes H_{s}(\xi)$.

Definition. For $n=3$ and $m \geqq 0$, set

$$
Q_{m}=\sum_{0 \leqq s \leqq r \leqq m ; r+s=m} \oplus P_{r, s}^{m} \oplus \sum_{0 \leqq s \leqq r \leqq m ; r+s=m+1} \oplus P_{r, s}^{m}
$$

Note that the total homogeneity of any polynomial in the first sum of (2.14) is exactly one less than that of any polynomial in the second.

THEOREM 4. For $n=3$ the space $Q_{m}$ is mapped by the restriction map to $C S\left(S^{2}\right)$ into the space $\mathscr{H}_{m}$. The restriction map commutes with the action of SO (3) on both spaces. Furthermore, the restriction map to $C S\left(S^{2}\right)$ has kernel 0 on $Q_{m}$ and carries $Q_{m}$ onto $\mathscr{H}_{m}$.

Proof. The demonstration repeats that of Theorem 2 until one considers the analogue of the equations (2.7) and (2.9). Now $q$ is no longer homogeneous, but as we have just remarked, it is the sum of two homogeneous pieces $q_{1}$ and $q_{2}$ that differ by 1 in their total homogeneity. Considering separately the terms of odd and even homogeneity in (2.7), we obtain two sets of equations analogous to (2.9). By the same argument as in the proof of Theorem 2 we show that both $q_{1}$ and $q_{2}$ are identically zero. 
Proof of Theorem 3. Suppose first that $n>3$, and let $q \in Q_{m}$. It is a homogeneous polynomial, of degree $p$ say. Since $S\left(V^{*}\right)=I\left(V^{*}\right) H\left(V^{*}\right)$ (see [8]),

$$
q=h+\sum r_{i} h_{i}
$$

where $h$ and each $h_{i}$ are harmonic polynomials and the $r_{i}$ are invariant polynomials. (We may assume $p>0$, since the case $p=0$ is trivial.)

Since SO $(n)$ acts by linear substitutions, it is easy to see that if $r$ is an invariant polynomial so is each of its homogeneous terms. Dually, if $D$ is an invariant differential operator, so is each term of fixed order (that is, each homogeneous part). Hence if $h$ is harmonic each term in the expression of $h$ as a sum of homogeneous polynomials must be annihilated by each invariant differential operator $D$.

Hence we may assume that in (2.15) $h$ is homogeneous of degree $p=$ degree $q$, while each of the $h_{i}$ appearing has homogeneity strictly less than $p$. Now we may apply the projection formula (1.10) to $q$. The resulting expression (2.12) becomes

$$
q(x, \xi)=d_{m} \int_{\text {so }(n)} h_{a}(x, \xi) \bar{\chi}_{m}(a) d a+0 .
$$

The integrals of the $h_{i}$ are 0 by the same argument as in Theorem 2, since their degree is lower than that of $q$. Finally, by applying any invariant differential operator under the integral sign, the right hand side of (2.16) is seen to be harmonic.

If $n=3$, we write $q=q_{1}+q_{2}$, where $q_{1}$ is homogeneous of odd degree and $q_{2}$ is homogeneous of even degree. The argument above may be carried out for each separately. (We observe that there are no invariant polynomials of odd degree. See $[25$, p. 32].)

Now let $h(x, \xi)$ be any SO (n)-harmonic polynomial. As we have shown above, each homogeneous term of $h$ is harmonic. Let us therefore assume that $h$ is homogeneous of degree $k$, and show that $h$ is a finite sum $h=\sum q_{m}$, where $q_{m} \in Q_{m}$. We begin by writing $h=\sum h_{m}+h_{1}$, where the sum is finite and

$$
h_{m}=d_{m} \int_{\mathrm{SO}(n)} h_{a} \bar{\chi}_{m}(a) d a=d_{m} \int_{\mathrm{So}(n)}\left(h_{m}\right)_{a} \bar{\chi}_{m}(a) d a,
$$

while for each $m$ the similar integral taken for $h_{1}$ vanishes.

Let us temporarily fix $m=\left(m_{1}, m_{2}, 0, \ldots, 0\right)$ and consider $h_{m}$. Substituting $t x$ for $x$ and $t \xi$ for $\xi$ in the last equation shows that $h_{m}$ is homogeneous of degree $k$. Its restriction to $C S\left(S^{n-1}\right)$ is in $\mathscr{H}_{m}$, and so there is a polynomial $q_{m}$ in $Q_{m}$ that agrees with $h_{m}$ on $C S\left(S^{n-1}\right)$. Let us denote the homogeneity of $q_{m}$ (or in the case $n=3$, that of the piece whose homogeneity has the same parity as $k$ ) by $p$. By Lemma 2 we must have $p \leqq k$. Since $h_{m}-q_{m}$ vanishes on $C S\left(S^{n-1}\right)$, we can, as in the proof of Theorem 2, obtain the equations

$$
\begin{aligned}
& A_{s}|x|^{2}+B_{s}|\xi|^{2}+C_{s} x \xi \\
& h_{m}-q_{m}=+A_{s-2}|x|^{2}+B_{s-2}|\xi|^{2}+C_{s-2} x \xi-A_{s}-B_{s}+\cdots \\
&+A_{k-2}|x|^{2}+B_{k-2}|\xi|^{2}+C_{k-2} x \xi-A_{k}-B_{k}+\cdots \\
&+A_{p-2}|x|^{2}+B_{p-2}|\xi|^{2}+C_{p-2} x \xi-A_{p}-B_{p}+\cdots
\end{aligned}
$$


If $p=k$, the argument following equations (2.9) may once again be applied to show that $h_{m}-q_{m}$ is the zero polynomial. If $p<k$, we use a similar recursion argument to obtain

$$
h_{m}=\left(A_{k-2}+\alpha_{k-2}\right)|x|^{2}+\left(B_{k-2}-\alpha_{k-2}\right)|\xi|^{2}+\left(C_{k-2}-\beta_{k-2}-\gamma_{k-2}\right) x \xi
$$

Since $S\left(V^{*}\right)$ is the direct sum of $H\left(V^{*}\right)$ and the ideal in $S\left(V^{*}\right)$ generated by $I_{+}\left(V^{*}\right)$, the invariant polynomials of positive degree, $h_{m}$ is the zero polynomial.

Finally, let us deal with the harmonic polynomial $h_{1}=h-\sum h_{m}$. It is homogeneous of degree $k$. Since for each $m$ and each $(x, \xi) \in C S\left(S^{n-1}\right)$

$$
d_{m} \int_{\text {So }(n)}\left(h_{1}\right)\left(a^{-1} x, a^{-1} \xi\right) \bar{\chi}_{m}(a) d a=0,
$$

the polỳnomial $h_{1}$ vanishes on $C S\left(S^{n-1}\right)$. We may now write equations analogous to (2.18) with $h_{m}-q_{m}$ replaced by $h_{1}$. A recursion argument like the one following equations (2.9) leads to an equation similar to (2.19), and so as above, $h_{1}$ is the zero polynomial. Thus any harmonic polynomial $h$ is a finite sum $h=\sum_{m} q_{m}$, with $q_{m} \in Q_{m}$.

The Corollary to Theorem 3 is proved by writing any harmonic polynomial $h$ as $h=\sum q_{m}$ with $q_{m} \in Q_{m}$. If $h$ vanishes on $C S\left(S^{n-1}\right)$, so does each $q_{m}$, since $\left\{\mathscr{H}_{m}\right\}$ is an orthogonal family. But by Theorem 2 , this means that each $q_{m}$ is identically 0 .

The proof of Proposition 1 proceeds with the use of some elementary notions and theorems in algebraic geometry (over the field of complex numbers), and of the celebrated Hilbert Nullstellensatz. We begin with a brief summary of the facts that will be used. If $K$ is a field, let $K\left[X_{1}, \ldots, X_{n}\right]$ denote the ring of polynomials in indeterminates $X_{1}, \ldots, X_{n}$.

Let $A$ be a ring with unit (usually a polynomial ring over a field, some quotient of one, or a localization of such). An ideal $I$ is prime in $A$ if and only if $A / I$ is an integral domain, and maximal if and only if $A / I$ is a field.

If $A$ is a quotient ring of $C\left[X_{1}, \ldots, X_{n}\right]$ and $m$ is a maximal ideal in $A$, then $A / m$ is isomorphic to $C$ (see $[19$, p. 5]).

If $I$ is an ideal in $K\left[X_{1}, \ldots, X_{n}\right]$, the locus (or variety) of $I$ in $K^{n}$ is the set of all points $\left(x_{1}, \ldots, x_{n}\right) \in K^{n}$ such that $f\left(x_{1}, \ldots, x_{n}\right)=0$ for each $f$ in $I$. A variety in $K^{n}$ is the locus in $K^{n}$ of some ideal of $K\left[X_{1}, \ldots, X_{n}\right]$. A variety is irreducible if it is not the union of proper varieties. Since $C$ is algebraically closed, the ideal $J$ of all polynomials that vanish on $V$, the locus of an ideal $I$ in $C\left[X_{1}, \ldots, X_{n}\right]$ is prime if and only if $V$ is irreducible.

The dimension $d$ of the locus of a prime ideal $I$ in $C\left[X_{1}, \ldots, X_{n}\right]$ is the transcendence degree of $C\left[X_{1}, \ldots, X_{n}\right] / I$ over $C$. This is equal to the dimension of the variety of $I$ as a manifold (at its nonsingular points).

If $I$ is an ideal in $C\left[X_{1}, \ldots, X_{n}\right]$ its radical $\sqrt{ } I$ is the set of all polynomials $f$ such that $f^{e} \in I$ for some positive integer $e$. By the Hilbert Nullstellensatz, if $I$ is any ideal in $C\left[X_{1}, \ldots, X_{n}\right]$, then the set of all polynomials $f$ in $C\left[X_{1}, \ldots, X_{n}\right]$ 
that vanish on the variety of $I$ is exactly the ideal $\sqrt{ } I$. (This uses the fact that $C$ is algebraically closed.)

For proofs and more discussion see [9, pp. 1-7], [13], or [27, pp. 160-168 of Vol. II].

We shall be interested in the polynomial ring $S=C\left[x_{1}, \ldots, x_{n}, \xi_{1}, \ldots, \xi_{n}\right]$. By $(p, q, \ldots)$ we shall mean the ideal in $S$ generated by $(p, q, \ldots)$. The next two lemmas will allow us to apply the Hilbert Nullstellensatz to any polynomial $q$ that vanishes on $C S\left(S^{n-1}\right)$.

LEMMA 3. The locus in $C^{2 n}$ of each of the ideals $I_{n}=\left(|x|^{2}-1,|\xi|^{2}-1, x \xi\right)$ and $J_{n}=\left(|x|^{2}-1,|\xi|^{2}-1\right)$ is connected.

Proof sketch. The complex locus of $I_{n}$ consists of points $\left(z_{1}, \ldots, z_{n}, w_{1}, \ldots, w_{n}\right)$ in $C^{2 n}$ satisfying the equations

$$
z_{1}^{2}+\cdots+z_{n}^{2}=1, \quad w_{1}^{2}+\cdots+w_{n}^{2}=1, \quad z_{1} w_{1}+\cdots+z_{n} w_{n}=0 .
$$

By setting $z_{j}=x_{j}+i y_{j}, w_{j}=\xi_{j}+i \eta_{j}$ we see that $C S\left(S^{n-1}\right)$ can be identified with the "real locus" of the three generating polynomials, i.e., those points $\left(z_{1}, \ldots, z_{n}\right.$, $w_{1}, \ldots, w_{n}$ ) such that for each $j=1, \ldots, n$ we have $y_{j}=0$ and $\eta_{j}=0$. Let us solve equations (2.20) for (say) $z_{1}, w_{1}$ and $w_{2}$ in terms of $z_{2}, \ldots, z_{n}, w_{3}, \ldots, w_{n}$. We can then define a curve in the complex locus starting from an arbitrary point $\left(z_{1}^{0}, \ldots, z_{n}^{0}, w_{1}^{0}, \ldots, w_{n}^{0}\right)$ to some point on the real locus as follows: we let the imaginary parts of $z_{2}, \ldots, z_{n}, w_{3}, \ldots, w_{n}$ tend to zero one at a time. Then we can let the numbers $x_{1}, \ldots, x_{n}, \xi_{3}, \ldots, \xi_{n}$ vary so that the resulting values of $z_{1}, w_{1}$ and $w_{2}$ are real. We omit details of the (double-valued) solutions and the precautions that must be taken (including relabeling if necessary) to avoid vanishing denominators. See [14] for these details. Since the real locus is connected, and any point in the complex locus can be connected by a path to a point on the real locus, the complex locus is connected.

The proof for $J_{n}$ is simpler.

LEMMA $4\left(^{2}\right)$. Let $n \geqq 3$. If a polynomial in $S$ vanishes on the real locus of

$$
\left(|x|^{2}-1,|\xi|^{2}-1, x \xi\right)
$$

in $R^{2 n}$, then it vanishes on the complex locus (2.20) of this ideal.

Proof. As in the proof of Lemma 3, we set $z_{j}=x_{j}+i y_{j}, w_{j}=\xi_{j}+i \eta_{j}$ and regard the real locus as those points such that $y_{j}=0, \eta_{j}=0$. Let $x^{0}=(\sqrt{ } 2 / 2, \sqrt{ } 2 / 2,0, \ldots, 0)$, $\xi^{0}=(\sqrt{ } 2 / 2,-\sqrt{ } 2 / 2,0, \ldots, 0)$, and solve the equations $(2.20)$ near $\left(x^{0}, \xi^{0}\right)$ to obtain

$$
\begin{aligned}
z_{1} & =\phi\left(z_{2}, \ldots, z_{n}\right), \\
w_{k} & =\psi_{k}\left(z_{2}, \ldots, z_{n}, w_{3}, \ldots, w_{n}\right), \quad k=1,2,
\end{aligned}
$$

$\left.{ }^{2}\right)$ The author would like to thank Dr. Pierre Samuel for suggesting the use of this lemma as well as for sending him the proof, due to A. Weil, given for Lemma 6. 
where $\phi, \psi_{1}$ and $\psi_{2}$ are analytic single-valued functions of their arguments for $\left|z_{2}-\sqrt{ } 2 / 2\right|<\delta,\left|z_{j}\right|<\delta,\left|w_{j}\right|<\delta, j \geqq 3$. It can be easily verified that if $z_{3}$ is close to $\sqrt{ } 2 / 2$ and real, and $z_{3}, \ldots, z_{n}, w_{3}, \ldots, w_{n}$ are close to zero and real, then the corresponding values of $z_{1}, w_{1}, w_{2}$ obtained from (2.21) will also be real.

If $f$ is any polynomial in $S$, then for $(z, w)$ close to $\left(x^{0}, \xi^{0}\right)$ we may write

$$
f(z, w)=f\left(\phi_{1}, z_{2}, \ldots, z_{n}, \psi_{1}, \psi_{2}, w_{3}, \ldots, w_{n}\right)=\sum_{\alpha, \beta} c_{\alpha}^{\beta} z_{2}^{\alpha_{2}} \cdots z_{n}^{\alpha_{n}} w_{3}^{\beta_{3}} \cdots w_{n}^{\beta_{n}}
$$

where $\alpha=\left(\alpha_{2}, \ldots, \alpha_{n}\right)$ and $\beta=\left(\beta_{3}, \ldots, \beta_{n}\right)$ are multi-indices. But each $c_{\beta}^{\alpha}$ in (2.22) can be computed by taking derivatives on the right side along real axes, i.e., along the $x_{2}, \ldots, x_{n}, \xi_{3}, \ldots, \xi_{n}$ axes. But if $f$ vanishes on the real locus, all such derivatives must be zero, by the remark at the end of the last paragraph. Hence if $f$ vanishes on the real locus it vanishes for all points on the complex locus distant (in $C^{2 n}$ ) from $\left(x^{0}, \xi^{0}\right)$ less than some positive number $\varepsilon$.

To complete the proof we note that the Jacobian

$$
J=\frac{\partial\left(|x|^{2}-1,|\xi|^{2}-1, x \xi\right)}{\partial\left(x_{1}, \ldots, x_{n}, \xi_{1}, \ldots, \xi_{n}\right)}
$$

has rank 3 at every point of the complex locus, so that the locus is an analytic manifold. Since this analytic manifold is connected (Lemma 3) any analytic function $f$ that vanishes on an open set of it vanishes identically on it.

At this point we can apply the Hilbert Nullstellensatz to obtain (2.7) with $q$ replaced by a positive integral power $q^{e}$. The following lemma allows us to assert that $e$ can be taken to be 1 , thus completing the proof of Proposition 1 .

LEMMA 5. The ideals $I_{n}$ and $J_{n}$ of Lemma 3 are each prime for $n \geqq 2$.

Proof. Since $V_{n}$, the locus in $C^{2 n}$ of $I_{n}$, is a connected analytic manifold (see proof of Lemma 4), it must be an irreducible variety, for if $V_{n}$ were reducible there would be two polynomials $f$ and $g$ such that $f g$ vanishes identically on $V_{n}$, but neither $f$ nor $g$ does. In this case, some nonempty open set in $V_{n}$ would be the union of the zero sets of $f$ and $g$, each of which, however, is $2 n-4$ dimensional. Since $V_{n}$ is irreducible, its ideal, which by the Hilbert Nullstellensatz is $\sqrt{ } I_{n}$, is prime. Lemma 6 together with the remarks after (2:23) shows that $I=\sqrt{ } I$.

The proof for $J_{n}$ is similar.

LemMA 6. Let $k$ be an algebraically closed field and I an ideal in $k\left[X_{1}, \ldots, X_{n}\right]$ whose radical $\sqrt{ } I$ is a prime ideal of dimension $d$. Suppose the Jacobian matrix $\left(\partial F_{j} / \partial X_{i}\right)$ of the generators $F_{1}, \ldots, F_{n-d}$ of $I$ is of rank $n-d$ at each zero of $I$ in $k^{n}$. Then $I=\sqrt{ } I$.

Proof. It must be shown that the affine coordinate ring $A=k\left[X_{1}, \ldots, X_{n}\right] / I$ $=k\left[x, \ldots, x_{n}\right]$ has no nilpotent elements. 
The $A$-module of differential forms $D=\Omega_{A / k}$ is the $A$-module defined by the generators $d x_{i}$ and the relations

$$
\sum_{i} \frac{\partial F}{\partial X_{i}}(x) d x_{i}=0
$$

for each $F \in I$. The hypothesis on the rank of the Jacobian implies that if $m$ is a maximal ideal of $A$, then $A / m \otimes_{A} D$ is a vector space of dimension $d$ over the field $A / m$. (Since $k$ is algebraically closed $A / m$ is actually isomorphic to $k$.)

For a fixed maximal ideal $m$ of $A$, let $A^{\prime}$ denote the local ring

$$
A_{m}=\{f / g \mid f, g \in A, g \in m\},
$$

and let $m^{\prime}$ denote its maximal ideal $m A^{\prime}$. Since $m$ is maximal $A / m=A^{\prime} / m^{\prime}(=k)$, and hence (by the localization of modules of differentials)

$$
A / m \otimes_{A} D=\left(A^{\prime} / m^{\prime}\right) \otimes_{A^{\prime}} \Omega_{A^{\prime} / k} .
$$

But there is a canonical isomorphism: $m / m^{\prime 2} \rightarrow\left(A^{\prime} / m^{\prime}\right) \otimes_{A^{\prime}} \Omega_{A^{\prime} / k}$. (See [6, exposé 17, No. 3, Theorem 5].) Hence the dimension of $m^{\prime} / m^{\prime 2}$ over $A^{\prime} / m^{\prime}$ is $d$, so that $A^{\prime}$ is (a regular local ring and in particular is) an integral domain [6, exposé 17 , No. 1 , Theorem 1 and Theorem 2].

Since each $A_{m}$ is a reduced ring, and the canonical homomorphism $A \rightarrow \Pi_{m \max } A_{m}$ is injective [2, Chapter II, Section 3, No. 3, Corollary 2 of Theorem 1] it follows that $A$ is a reduced ring.

REMARK. The proof of Lemma 6 given above involves the notion of a regular local ring. For a discussion see either [6] or [27, Vol. II, pp. 301-302].

LEMMA 7. For $n \geqq 3$ the ideal $\left(|x|^{2},|\xi|^{2}\right)$ is prime in $S$.

Proof. This follows from the fact that $\left(|x|^{2}\right)$ is prime in $C\left[x_{1}, \ldots, x_{n}\right]$ and $\left(|\xi|^{2}\right)$ is prime in $C\left[\xi_{1}, \ldots, \xi_{n}\right]$. See $[13$, p. 86].

Proof of Proposition 2. The ideal the three elements generate is easily seen to be contained in the kernel. Let $(A, B, C)$ lie in the kernel. If $C \neq 0$ then $C x \xi \in$ $\left(|x|^{2},|\xi|^{2}\right) \subseteq S$, while $x \xi$ does not lie in this ideal (since it is linear in $x_{1}, \ldots, x_{n}$ and in $\left.\xi_{1}, \ldots, \xi_{n}\right)$. But by Lemma 7 the ideal $\left(|x|^{2},|\xi|^{2}\right)$ is prime in $S$, and so $C=-\beta|x|^{2}-\gamma|\xi|^{2}$ for $\beta, \gamma \in S$.

Hence $(A-\beta x \xi)|x|^{2}+(B-\gamma x \xi)|\xi|^{2}=0$, and since $|x|^{2}$ is irreducible in $S$, and $|\xi|^{2}$ does not divide it,

$$
B-\gamma x \xi=-\alpha|x|^{2} \quad \text { for } \alpha \in S .
$$

Thus $(A-\beta x \xi)|x|^{2}=\alpha|x|^{2}|\xi|^{2}$ and so $A=\alpha|\xi|^{2}+\beta x \xi$.

III. To construct singular operators we shall start with differential operators, and compose them with suitable powers of $\Lambda^{-1}$, where $-\Lambda^{2}$ approximates the Laplacian on $S^{n-1}$. These powers of $\Lambda^{-1}$ will be considered in connection with the function spaces $L_{k}^{p}\left(S^{n-1}\right)$ for $1<p<\infty$ and $k$ any integer. See [21] for a definition 
of these spaces in terms of a partition of unity $\left\{\phi_{i}\right\}$. For each $k, L_{k}^{p}=L_{k}^{p}\left(S^{n-1}\right)$ is a reflexive Banach space that is isomorphic to $L^{p}\left(S^{n-1}\right)$. If $k \geqq 0$, then the space of $C^{\infty}$ functions is dense in $L_{k}^{p}$. It is not hard to show that any norm for $L_{k}^{p}$ arising from a partition of unity as in [21] is equivalent to the norm

$$
\|f\|_{p, k}=\left\{\sum_{0 \leqq n \leqq k}\left\|D_{i_{1}}, \ldots, D_{i_{h}} f\right\|_{p}^{p}\right\}^{1 / p},
$$

where the summation is over all $h$-fold iterates of standard basis elements $D_{i j}=x_{i}\left(\partial / \partial x_{j}\right)-x_{j}\left(\partial / \partial x_{i}\right)$ of $S_{n}$, the Lie algebra of SO $(n)$. The measure used in defining $\|f\|_{p, 0}=\|f\|_{p}$ is $d \sigma$, the (unique) rotation invariant measure normalized so that $\int_{S^{n-1}} d \sigma=\omega_{n-1}$, the surface area of $S^{n-1}$.

Definition. Let $r$ be a nonnegative integer. We say that $T$ is smoothing of order $r$ on $L^{p}\left(S^{n-1}\right)$ if for all integers $k, T$ is continuous: $L_{k}^{p}\left(S^{n-1}\right) \rightarrow L_{k+r}^{p}\left(S^{n-1}\right)$. If no particular $p$ is mentioned it is assumed that this holds for each $p$ with $1<p<\infty$.

Proposition 3. Let $T: C^{\infty}\left(S^{n-1}\right) \rightarrow C^{\infty}\left(S^{n-1}\right)$ be an operator that commutes with the action $L_{a}$ of $\mathrm{SO}(n)$ on $C^{\infty}\left(S^{n-1}\right)$. Suppose that $T$ is bounded: $L^{p} \rightarrow L^{p}$. Then

(a) For any $D \in S_{n}, T D=D T$ on $C^{\infty}\left(S^{n-1}\right)$;

(b) $T$ is smoothing of order 1 if and only if for any $D \in S_{n} D T$ is bounded: $L^{p} \rightarrow L^{p}$;

(c) $T$ is smoothing of order $r \geqq 1$ if and only if for any $r$-fold iterate $D$ of elements of $S_{n}, D T$ is bounded: $L^{p} \rightarrow L^{p}$.

Proof sketch. Conclusion (a) follows immediately from

$$
(D f)(x)=\lim _{t \rightarrow 0} t^{-1}[f(\exp t D x)-f(x)],
$$

since $T$ commutes with rotations.

If $T$ is bounded: $L_{k}^{p} \rightarrow L_{k+1}^{p}$ for each $k$, then taking $k=0$ and using (3.1) in the definition of a smoothing operator shows $D T$ is bounded $L^{p} \rightarrow L^{p}$. For the converse we use the fact that if $h \leqq k+1$,

$$
D_{i_{1}} D_{i_{2}} \cdots D_{i_{h}} T f=D_{i_{1}} T\left(D_{i_{2}} \cdots D_{i_{h}}\right) f .
$$

Thus $\left\|D_{i_{1}} T\left(D_{i_{2}} \cdots D_{i_{h}} f\right)\right\|_{p} \leqq C_{p}\left\|D_{i_{2}} \cdots D_{i_{h}} f\right\|_{p} \leqq C_{p}\|f\|_{p, k}$, and summing over $h$-fold iterates with $0 \leqq h \leqq k+1$ yields the desired estimate. Part (c) is proved analogously.

Let $P_{r}(t)$ denote the Poisson kernel

$$
\frac{1}{\omega_{n-1}}\left(1-r^{2}\right)\left(1-2 r t+r^{2}\right)^{-n / 2}
$$

for $S^{n-1}$. We define the operator $\Lambda^{-1}$ on $C^{\infty}\left(S^{n-1}\right)$ by

$$
\left(\Lambda^{-1} f\right)(x)=\int_{S^{n-1}} K(x \cdot y) f(y) d y,
$$


where

$$
K(t)=-\int_{0}^{1} P_{r}(t) d r
$$

Proposition 4. The operator $\Lambda^{-1}$ is bounded: $L^{p}\left(S^{n-1}\right) \rightarrow L^{p}\left(S^{n-1}\right)$ for $1 \leqq p \leqq \infty$. If $Y_{s} \in H_{s}$, the spherical harmonics of degree $s$, then

$$
\Lambda^{-1} Y_{s}=-Y_{s} /(s+1)
$$

Proof. The first statement follows from Hölder's inequality and the fact that for each $x, K(x \cdot y)$ is an $L^{1}\left(S^{n-1}\right)$ function of $y$ such that $K(a x \cdot a y)=K(x \cdot y)$. (In fact, $\Lambda^{-1}$ is given as a convolution on the sphere, regarded as a homogeneous space of $\mathrm{SO}(n)$, with an $L^{1}$ function.)

If $Y_{s}$ is in $H_{s}$, then

$$
\int_{S^{n-1}} P_{r}(x \cdot y) Y_{s}(y) d y=r^{s} Y_{s}(x)
$$

(See [3] or [22] for a discussion of spherical harmonics in $n$ dimensions.) Interchanging the order of integration and letting $\rho \rightarrow 1$ in the formula

$$
\int_{0}^{\rho}\left\{\int_{s^{n-1}} P_{r}(x \cdot y) Y_{s}(y) d y\right\} d \rho=\frac{\rho^{s}}{s+1} Y_{s}
$$

that follows from (3.7) establishes (3.6).

Proposition 5. For $1<p<\infty$ and $k$ an integer, the operator $\Lambda^{-1}$ is bounded: $L_{k}^{p}\left(S^{n-1}\right) \rightarrow L_{k+1}^{p}\left(S^{n-1}\right)$.

Proof. By Proposition 3, it is sufficient to show that if $D_{i j}$ is a standard basis element of $S_{n}$, then $D_{i j} \Lambda^{-1}$ is bounded: $L^{p} \rightarrow L^{p}$. Since $L_{a} \Lambda^{-1}=\Lambda^{-1} L_{a}$ and

$$
L_{a} D_{i j} L_{a}^{-1}=\operatorname{adj}_{a}\left(D_{i j}\right)=\sum_{k<l} a_{k i} a_{l j} D_{k l}
$$

we have

$$
L_{a} D_{i j} \Lambda^{-1} L_{a}^{-1}=\sum_{k<l} a_{k i} a_{l j} D_{k l} \Lambda^{-1}
$$

Let $U$ be any open set containing $N=(0, \ldots, 0,1)$, and let $f$ be any $C^{\infty}\left(S^{n-1}\right)$ function supported in $a U=\{a x \mid x \in U\}$. Then for any basis element $D_{i j}$ of $S_{n}$ and $f_{a}=L_{a} f$ (which is supported in $U$ ),

$$
\begin{aligned}
\left\|D_{i j} \Lambda^{-1} f\right\|_{p} & =\left\|L_{a} D_{i j} \Lambda^{-1} L_{a}^{-1} f_{a}\right\|_{p}=\left\|\sum_{k<l} a_{k i} a_{l j} D_{k l} \Lambda^{-1} f_{a}\right\|_{p} \\
& \leqq \frac{n(n-1)}{2} \operatorname{Max}_{k<l}\left\|D_{k l} \Lambda^{-1} f_{a}\right\|_{p} .
\end{aligned}
$$

Hence it is sufficient to show that $\left\|\Lambda^{-1} f\right\|_{p, 1} \leqq C\|f\|_{p}$ for $C^{\infty}$ functions $f$ supported in some small open set of $S^{n-1}$

$$
U_{\varepsilon}=\left\{\left(x_{1}, \ldots, x_{n}\right) \mid \begin{array}{l}
x_{1}^{2}+\ldots+x_{n}^{2}=1 \\
1-\varepsilon<x_{n} \leqq 1
\end{array}\right\}
$$


where $\varepsilon>0$ can be any fixed positive number. In fact, we shall prove the corresponding inequality for local coordinates $\bar{x}=\left(x_{1}, \ldots, x_{n-1}\right) \in E_{n-1}$ for $U_{\varepsilon}$ gotten by projecting a point $x \in U_{\varepsilon}$ onto the equatorial plane $x_{n}=0$. If $t=x \cdot y$ $=x_{1} y_{1}+\cdots+x_{n} y_{n}$, we may write $t=t(\bar{x}, \bar{y})$ for $|\bar{x}|^{2}=x_{1}^{2}+\cdots+x_{n-1}^{2}$ and $|\bar{y}|^{2}=y_{1}^{2}+\cdots+y_{n-1}^{2}$ small (this makes $t$ close to 1 ).

LEMMA 8. The kernel $K(t)$ given by (3.5) may be written as

$$
\begin{aligned}
K(t)= & a_{n}(1-t)^{-(n-2) / 2}-\omega_{n-1}^{-1} \int_{0}^{1}\left(1-r^{2}\right)\left(1-r^{n-4}\right)\left(1-2 r t+r^{2}\right)^{-n / 2} d r \\
& +\left(\frac{n-4}{n-2}\right) \omega_{n-1}^{-1} \int_{0}^{1} r^{n-4}\left(1-2 r t+r^{2}\right)^{(2-n) / 2} d r
\end{aligned}
$$

Proof. Differentiation of both sides establishes the following identity given in [16], valid for $\gamma>-1$ :

$$
\begin{aligned}
\int_{0}^{r} t^{2 \lambda}\left(1-t^{2}\right)\left(1-2 a t+t^{2}\right)^{-\lambda-2} d t= & \frac{r^{2 \lambda+1}}{\lambda+1}\left(1-2 a r+r^{2}\right)^{-\lambda-1} \\
& -\frac{\lambda}{\lambda+1} \int_{0}^{r} t^{2 \lambda}\left(1-2 a t+t^{2}\right)^{-\lambda-1} d t .
\end{aligned}
$$

Writing

$$
P_{r}(t)=\omega_{n-1}^{-1} r^{n-4}\left(1-r^{2}\right)\left(1-2 r t+r^{2}\right)^{-n / 2}+\omega_{n-1}^{-1}\left(1-r^{n-4}\right)\left(1-r^{2}\right)\left(1-2 r t+r^{2}\right)^{-n / 2},
$$

integrating from 0 to 1 with respect to $r$ and applying (3.11) yields (3.10), with $a_{n}=-2^{(2-n) / 2} \omega_{n-1}^{-1}(2 / n-2)$.

Let us rewrite (3.10) as

$$
K(t)=a_{n}(1-t)^{-(n-2) / 2}+A(t)+B(t) .
$$

For $t \neq 1$ both kernels $A(t)$ and $B(t)$ can be differentiated under the integral sign with any operator $D_{i j}=x_{i}\left(\partial / \partial x_{j}\right)-x_{j}\left(\partial / \partial x_{i}\right)$, or a similar one in the $y$ variables. Since such operators span the tangent plane at any point of $U_{\varepsilon}$, we may estimate the gradient $\nabla_{\bar{x}, \bar{y}}$ of $A$ or $B$ (with respect to differentiation in local coordinates) in terms of derivatives given by the Lie algebra. A simple estimate of such derivates shows that if $r(\bar{x}, \bar{y})$ denotes one of the kernels $A(t(\bar{x}, \bar{y}))$ or $B(t(\bar{x}, \bar{y}))$, then $r(\bar{x}, \bar{y})$ is $C^{\infty}$ for $\bar{x} \neq \bar{y}$ and for $|\bar{x}|$ and $|\bar{y}|$ small

$$
r(\bar{x}, \bar{y})=O\left(|\bar{x}-\bar{y}|^{-n+3}\right), \quad \nabla_{\bar{x}, \bar{y}} r(\bar{x}, \bar{y})=O\left(|\bar{x}-\bar{y}|^{-n+2}\right) .
$$

Now if $(R f)(\bar{x})=\int r(\bar{x}, \bar{y}) f(\bar{y}) d \bar{y}$, it follows from the mean-value theorem together with (3.13) and Lebesgue's dominated convergence theorem that $\left(\partial / \partial x_{i}\right) R f$ $(i=1, \ldots, n-1)$ is given by a kernel $k(\bar{x}, \bar{y})$ satisfying

$$
\begin{aligned}
& \int|k(\bar{x}, \bar{y})| d \bar{x}=O(1) \text { uniformly in } \bar{y}, \\
& \int|k(\bar{x}, \bar{y})| d \bar{y}=O(1) \text { uniformly in } \bar{x} .
\end{aligned}
$$


Hence each operator $\left(\partial / \partial x_{i}\right) R$ is bounded: $L^{p}\left(U_{\varepsilon}\right) \rightarrow L^{p}\left(U_{\varepsilon}\right)$, and hence, $R f$ is bounded: $L^{p}\left(U_{\varepsilon}\right) \rightarrow L_{1}^{p}\left(U_{\varepsilon}\right)$. See [15, p. 59].

REMARK. In the proof of Proposition 8 we shall show that $A(t)$ and $B(t)$ in (3.12) give operators that are actually smoothing of order 2. (The proof will use the result that $\Lambda^{-1}$ is smoothing of order 1.)

To complete the proof of Proposition 6 we now show that the first term in (3.12), which we denote by $K_{1}(t)$, gives a bounded operator: $L^{p}\left(U_{\varepsilon}\right) \rightarrow L_{1}^{p}\left(U_{\varepsilon}\right)$. In fact

$$
K_{1}(t(\bar{x}, \bar{y}))=-(2 /(n-2)) \omega_{n-1}^{-1} Q(x, y)|\bar{x}-\bar{y}|^{-n+2},
$$

where

$$
Q(\bar{x}, \bar{y})=2^{(2-n) / 2}(1-t)^{(2-n) / 2}|\bar{x}-\bar{y}|^{n-2}
$$

is $C^{\infty}$ for $\bar{x} \neq \bar{y}$ and satisfies

$$
\lim _{\bar{y} \rightarrow \bar{x}} Q(\bar{x}, \bar{y})=x_{n}^{n-2} .
$$

Now the function $Q^{2 /(n-2)}=\frac{1}{2}|\bar{x}-\bar{y}|^{2}(1-t)^{-1}$ is for $|\bar{x}-\bar{y}|<\frac{1}{2}$ an analytic function of the variables $z_{j}=x_{j}-y_{j}(j=1, \ldots, n-1)$. By using the Riemann removable singularities theorem for the function $\phi_{j}\left(z_{j}\right)$ obtained by setting all the $z$ 's other than $z_{j}$ equal to 0 we see that in fact $Q^{2 /(n-2)}$ is an analytic function whose real part is bounded away from 0 for $|\bar{x}|<1-\delta$. Hence $Q(\bar{x}, \bar{y})$ is actually $C^{\infty}$ for all $\bar{x}, \bar{y}$. Let us write

$$
\begin{aligned}
Q(\bar{x}, \bar{y})=Q^{\prime}(\bar{x}, \bar{x}-\bar{y})= & x_{n}^{n-2}+a_{1}(\bar{x})|\bar{x}-\bar{y}|+a_{2}(\bar{x})|\bar{x}-\bar{y}|^{2}+\cdots \\
& +a_{n-2}(\bar{x})|x-y|^{n-2}+b(x, y)|\bar{x}, \bar{y}|^{n-1},
\end{aligned}
$$

where each $a_{j}$ and $b$ are bounded $C^{\infty}$ functions with compact support. Substituting this expression into (3.15) and using (3.5) we see that since $\omega_{n-1}^{-1}=\frac{1}{2} \Gamma\left(\frac{1}{2} n\right) \pi^{-(1 / 2) n}$,

$$
K_{1}(t(\bar{x}, \bar{y}))=x_{n}^{n-2}(2-n)^{-1} \Gamma(n / 2) \pi^{-n / 2}|\bar{x}-\bar{y}|^{-n+2}+r,
$$

where $r(\bar{x}, \bar{y})$ gives rise to an operator $R$ that is bounded: $L^{p}\left(U_{\varepsilon}\right) \rightarrow L_{2}^{p}\left(U_{\varepsilon}\right)$. (See [15, p. 59].) The first term in (3.19) gives in coordinates $\bar{x}$ for $U_{\varepsilon}$ the operator $x_{n}^{n-2} \Lambda_{E}^{-1}$ defined by

$$
\begin{aligned}
\Lambda_{E} f & =i \sum_{j=1}^{n-1}\left(\frac{\partial}{\partial x_{j}}\right) R_{j} f, \\
\left(R_{j} f\right)(\bar{x}) & =-i \Gamma\left(\frac{n}{2}\right) \pi^{-n / 2} \lim _{\varepsilon \rightarrow 0} \int_{|\bar{x}-\bar{y}|>\varepsilon} f(\bar{y})\left(\bar{x}_{j}-\bar{y}_{j}\right)|\bar{x}-\bar{y}|^{-n} d y .
\end{aligned}
$$

(See [21, p. 662].)

Since $U_{\varepsilon}$ has compact closure, $\Lambda_{\bar{E}}^{-1}$ is bounded: $L^{p}(V) \rightarrow L_{1}^{p}(V)$, where $V$ is the domain of the coordinates $\bar{x}$ for $U_{\varepsilon}$. This concludes the proof.

We remark that a simpler proof would show that $R$ given by $r$ in (3.19) is smoothing of order 1 on $U_{\varepsilon}$, but for the proof of Proposition 8 we need the fact that it is smoothing of order 2. Also, it is possible to arrive at a similar inequality 
without using complex variable theory by suitable use of Taylor's theorem after transforming the expression (3.16) for $Q(\bar{x}, \bar{y})$.

The invariant Laplacian on $S^{n-1}$ is given by $\Delta=\sum_{i<j} D_{i j}^{2}$. In terms of its action on spherical harmonics, $\Delta Y_{s}=-s(s+n-2) Y_{s}$ if $Y_{s} \in H_{s}$. (See [7, pp. 385-389 and p. 397] as well as [3] and [26, pp. 61-63].) Using $\Delta$ we can define the inverse to $\Lambda^{-1}$ by

$$
\Lambda=-\Delta \Lambda^{-1}+(n-4) I+(3-n) \Lambda^{-1} .
$$

A simple computation shows that if $Y_{s} \in H_{s}$

$$
\Lambda Y_{s}=-(s+1) Y_{s}
$$

so that $\Lambda$ and $\Lambda^{-1}$ are inverse to each other on $C^{\infty}\left(S^{n-1}\right)$.

But it follows from Proposition 5 and the fact that $\Delta$ is bounded: $L_{k+2}^{p} \rightarrow L_{k}^{p}$ that $\Lambda$ is bounded: $L_{k+1}^{p} \rightarrow L_{k}^{p}$ for $k \geqq 0$. Since the dual of $L_{k}^{p}$ is $L_{-k}^{q}$ for $1<p<\infty$ and $q=p /(p-1)$, we obtain analogous results for $k<0$. We may summarize by stating

Proposition 6. For all integral $k$ and $1<p<\infty$, the map $\Lambda^{-1}$ is an isomorphism: $L_{k}^{p}\left(S^{n-1}\right) \rightarrow L_{k+1}^{p}\left(S^{n-1}\right)$. Its inverse is $\Lambda$ given by (3.21) or (3.22).

Thus $\Lambda^{-1}$ bears a good analogy to $\left(1+\Lambda_{E}\right)^{-1}$, where $\Lambda_{E}^{-1}$ is defined by (3.20).

DeFINITION. The multiplier on spherical harmonics determined by the sequence $\left\{\lambda_{s}\right\}_{s=0}^{\infty}$ of complex numbers is the operator $T$ defined by $T Y_{s}=\lambda_{s} Y_{s}$ if $Y_{s} \in H_{s}$.

We shall often denote this operator by $\left(\lambda_{s}\right)$. Thus,

$$
\Lambda^{-1}=-1 /(s+1), \quad \Delta=(-s(s+n-2)) .
$$

Proposition 7. The multiplier on spherical harmonics $(1 /(s+1)-1 /(s+2))$ is bounded: $L_{k}^{p}\left(S^{n-1}\right) \rightarrow L_{k+2}^{p}\left(S^{n-1}\right)$ for all integral $k$ and all complex $\lambda$.

Proof. We consider only the case $k=0$. The case $k \geqq 0$ follows from a duality argument. Expanding $1 /(x+\lambda)$ in a power series in $1 /(x+1)$ and setting $x=s$ gives

$$
\frac{1}{s+2}-\frac{1}{s+1}=(s+1)^{-2} \sum_{j=0}^{\infty}(1-\lambda)^{j+1}(s+1)^{-j}
$$

with convergence absolute and uniform for fixed $\lambda$ and $s \geqq 1$. We may restrict consideration to $s \geqq 1$ since the operator sending $f$ to $\int_{S^{n-1}} f d \sigma$ is smoothing of all orders. In view of Proposition 5 it is enough to show for $s \geqq 1$ the infinite series in (3.23) gives rise to a bounded operator on $L^{p}\left(S^{n-1}\right)$. It is sufficient to show that for some integer $N$ (which may depend on $\lambda$ ) $\sum_{N+1}^{\infty}(1-\lambda)^{j+1}(1 /(s+1))^{j}$ gives rise to a bounded operator on $L^{p}$.

Let $P_{s}(x \cdot y)$ be the zonal harmonic of degree $s$ normalized so that $P_{s}(1)=1$. (See [3] or [7] for a discussion.) Then the kernel $\left(1 / \omega_{n-1}\right) \sum_{0}^{\infty} d_{s} c_{s} P_{s}(x \cdot y)$ gives rise to the multiplier on spherical harmonics determined by the sequence $\left\{c_{s}\right\}$. (Here $d_{s}$ denotes the dimension of $H_{s}$.) For example, setting $c_{s}=r^{s}$ yields the Poisson kernel. 
If $c_{s}=O\left(s^{-n / 2}\right)$, then the kernel written down above is an $L^{2}$ function of $y$ for each fixed $x$. This follows from $\int P_{s}^{2} d x=1 / d_{s}$, Parseval's theorem, and the estimate $d_{s}=O\left(s^{n-2}\right)$. (See [5] for the last.) If we choose $N$ so large that uniformly in $s \geqq 1$

$$
\left|\sum_{N+1}^{\infty}(1-\lambda)^{j+1}(s+1)^{-j}\right| \leqq c_{\lambda} s^{-n / 2},
$$

then the operator it gives rise to will be given by a kernel $K_{N}(x \cdot y)$ that is an $L^{2}$ function of $y$ for each $x$ and invariant under rotations, and hence is bounded on $L^{p}$.

We are now in a position to obtain a more precise expression for $\Lambda^{-1}$ in local coordinates $\bar{x}$ for $U_{1 / 2}=\left\{x \in S^{n-1} \mid \frac{1}{2}<x_{n} \leqq 1\right\}$.

Proposition 8. In local coordinates $\bar{x}$ for $U_{1 / 2}$,

$$
\left(\Lambda^{-1} f\right)(\bar{x})=x_{n}^{n-2}\left(\Lambda_{E}^{-1} f\right)+(R f)(\bar{x}),
$$

where for each integral $k, R$ is bounded: $L_{k}^{p}\left(U_{1 / 2}^{\prime}\right) \rightarrow L_{k+2}^{p}\left(U_{1 / 2}\right)$ and $\Lambda_{E}^{-1}$ is defined by (3.20).

Proof. In view of the expression (3.19) for $K_{1}(t(\bar{x}, \bar{y}))$ and the remark made after (3.20), it is sufficient to prove that the last two terms in (3.12) give bounded operators: $L_{k}^{p}\left(U_{1 / 2}\right) \rightarrow L_{k+2}^{p}\left(U_{1 / 2}\right)$. We shall show that they give bounded operators: $L_{k}^{p}\left(S^{n-1}\right) \rightarrow L_{k+2}^{p}\left(S^{n-1}\right)$. As in the proof of Proposition 7 we may restrict consideration to the case $k=0$.

Integrating $A(t)$ in (3.12) against $Y_{s} \in H_{s}$ shows that $A(t)$ gives rise to the multiplier on spherical harmonics $(1 /(s+n-3)-1 /(s+1))$, which has the desired boundedness by Proposition 7. The operator arising from $B(t)$ is treated by writing

$$
\left(1-2 r t+r^{2}\right)^{(2-n) / 2}=\sum_{s=0}^{\infty} \frac{n-2}{2 s+n-2} d_{s} r^{s} P_{s}(t),
$$

where $P_{s}(t)$ is as in the proof of Proposition 7. (If $\lambda=(n-2) / 2$, then

$$
P_{s}(t)=P_{s}^{\lambda}(t) / P_{s}^{\lambda}(1),
$$

where $\left(1-2 r t+r^{2}\right)^{-\lambda}=\sum_{0}^{\infty} r^{s} P_{s}^{\lambda}(t)$. See [5, p. 903] and [3].) Multiplying (3.25) by $r^{n-4}$ and integrating from 0 to 1 with respect to $r$ gives an $L^{1}\left(S^{n-1}\right)$ function of $y$ for each fixed $x$. It has the expansion

$$
\sum_{0}^{\infty} \frac{n-2}{2 s+n-2} \frac{1}{s+n-3} d_{s} P_{s}(t)
$$

Thus integration against $B(t)$ yields the multiplier on spherical harmonics

$$
\frac{n-4}{n-2}\left(\frac{1}{2 s+n-2}\right)\left(\frac{1}{s+n-3}\right)
$$

which is bounded: $L_{k}^{p}\left(S^{n-1}\right) \rightarrow L_{k+2}^{p}\left(S^{n-1}\right)$ by Propositions 7 and 5 .

As a corollary of Proposition 8 we have 
Proposition 9. Let $D$ be a differential operator of order $s$ on $S^{n-1}$ with $C^{\infty}$ coefficients. Then

$$
i^{s} D \circ \Lambda^{-s}=A+S,
$$

where $A \in C_{\infty}^{\infty}\left(S^{n-1}\right)$ and $S$ is a smoothing operator.

Proof. We may assume the coefficients of $D$ have support in an open set of $S^{n-1}$ having arbitrarily small diameter. Since $\Lambda^{-1}$ commutes with rotations, we may assume this set is $U_{\varepsilon}$ as in Proposition 8. We write

$$
\left(i^{s} D \circ \Lambda^{-s}\right) f(\bar{x})=\sum_{|\alpha| \leqq s} a_{\alpha}(\bar{x})\left[D^{\alpha}\left(x_{n}^{n-2} \Lambda_{E}^{-1}\right) f\right](\bar{x})+R f(\bar{x}),
$$

where the notation is as before and $\alpha=\left(\alpha_{1}, \ldots, \alpha_{n-1}\right)$ is a multi-index. Using the Leibnitz rule on the terms in brackets we may express $i^{s} D \circ \Lambda^{-s}$ as the sum of a Euclidean singular integral operator and a remainder that smooths.

IV. In this chapter we prove the classification and decomposition theorems for singular operators on $L^{p}\left(S^{n-1}\right)$. The main tool is a linear map $q \rightarrow T_{q}$ from the space of SO $(n)$-harmonic polynomials to the $C_{\infty}^{\infty}\left(S^{n-1}\right)$ operators. It is constructed in such a way that

$$
\sigma\left(T_{q}\right)=\left.q\right|_{C S}, \quad L_{a} T_{q} L_{a}^{-1}=T_{q_{a}},
$$

where $q_{a}(x, \xi)=q\left(a^{-1} x, a^{-1} \xi\right)$. The fact that for each harmonic polynomial $q$ the operator $T_{q}$ is actually in $C_{\infty}^{\infty}\left(S^{n-1}\right)$ follows from Proposition 9. Let us assume that this map $q \rightarrow T_{q}$ has already been defined. Using Theorem 3 we see that for each dominant weight of the form $m=\left(m_{1}, m_{2}, 0, \ldots, 0\right)$ we obtain a family $\mathcal{O}_{m}=\left\{T_{q} \mid q \in Q_{m}\right\}$. The equations (4.1) show that in fact $q \rightarrow T_{q}$ gives an equivalence between the action of SO $(n)$ on $Q_{m}$ and that given on $\mathcal{O}_{m}$ by $T \rightarrow L_{a} T L_{a}^{-1}$. This representation of $\mathrm{SO}(n)$ on $\mathcal{O}_{m}$ is not irreducible, but we may use the decomposition (2.6) of $Q_{m}$ into irreducible subspaces to write

$$
\mathcal{O}_{m}=\sum_{r+s=m_{1}+\left|m_{2}\right| ;\left|m_{2}\right| \leqq r \leqq m_{1}} \oplus V_{r, s}^{m}
$$

where $V_{r, s}^{m}=\left\{T_{q} \mid q \in P_{r, s}^{m}\right\}$ is invariant and irreducible under the action of SO (n) (with the suitable modification for $n=3$, using (2.15)).

Definition. By a system of operators on $L^{p}\left(S^{n-1}\right)$ (or some other Banach space) we shall mean a (complex) vector space $V$ of such operators $T$. The systems $V_{r, s}^{m}$ defined above will be called the canonical systems of singular operators.

DEFINITION. We shall say that a system $V$ transforms according to a representation $R$ of $\mathrm{SO}(n)$ on $V$ if for each $a \in \mathrm{SO}(n)$ and $T \in V$

$$
L_{a} T L_{a}^{-1}=R_{a} T \text {. }
$$

If $\left\{V_{i}\right\}$ is a (finite or infinite) collection of systems of $C_{\infty}^{\infty}\left(S^{n-1}\right)$ operators transforming according to (possibly inequivalent) representations $R_{\mathfrak{i}}$ of $\mathrm{SO}(n)$, we shall 
say that $\left\{V_{i}\right\}$ is a collection of independent systems if $T_{1}+\cdots+T_{k}$ (with $T_{i} \in V_{i}$ ) smooths implies that each $T_{i}$ smooths.

THEOREM 5. Any singular operator $T$ in $C_{\infty}^{\infty}\left(S^{n-1}\right)$ can be decomposed into a sum

$$
T=\sum_{m} \sum_{i=1}^{s(m)} T_{m, i}
$$

where $T_{m, i}=T_{m, i}^{\prime}+S_{m, i}$, with $T_{i}^{m}$ an element of one of the canonical systems $V_{r, s}^{m}$ and $S_{m, i}$ a smoothing operator.

The summation is over all dominant weight vectors of the form

$$
m=\left(m_{1}, m_{2}, 0, \ldots, 0\right) ;
$$

for such $m, s(m)=m-\left|m_{2}\right|+1$ if $n \geqq 4,=2 m+1$ if $n=3$.

THEOREM 6. If $n \geqq 4$ and $m=\left(m_{1}, m_{2}, 0, \ldots, 0\right)$ is a dominant weight there are $m_{1}-\left|m_{2}\right|+1$ independent systems of operators in $C_{\infty}^{\infty}\left(S^{n-1}\right)$ transforming according to the irreducible representation $R^{m}$ of $\mathrm{SO}(n)$; if $m$ is not of this form there are no such systems.

If $n=3$ the number for $R^{m}$ is $2 m+1$.

Remark. The first equation in (4.1) together with Theorem 2 shows that for fixed $m=\left(m_{1}, m_{2}, 0, \ldots, 0\right)(n \geqq 4)$ the systems $V_{r, s}^{m} \subset \mathcal{O}_{m}$ are in fact $m_{1}-\left|m_{2}\right|+1$ independent systems of $C_{\infty}^{\infty}\left(S^{n-1}\right)$ operators. Of course, a different decomposition of $Q_{m}$ into irreducible subspaces could be used to define $m_{1}-\left|m_{2}\right|+1$ independent systems. (Suitable modifications hold for $n=3$.) But the space $\mathcal{O}_{m}$ can be invariantly characterized, modulo smoothing operators.

Before giving the proofs of Theorems 5 and 6 we construct the map $q \rightarrow T_{q}$ satisfying (4.1). We begin by defining the operators $R_{j}$ and $X_{j}$ and computing their symbols. For more details see $\S 4$ of [14].

The first-order differential operators $D_{1}, \ldots, D_{n}$ on $S^{n-1}$ are defined by extension of functions to $E_{n}$ : for $f \in C^{\infty}\left(S^{n-1}\right)$

$$
\left(D_{j} f\right)(x)=|x| \frac{\partial}{\partial x_{j}} f\left(\frac{x}{|x|}\right)
$$

where $\partial / \partial x_{j}$ denotes differentiation in the $j$ th direction in $E_{n}$. We note that the $D_{j}$ inherit the transformation properties of the $\partial / \partial x_{j}$ :

$$
L_{a} D_{j} L_{a}^{-1}=\sum_{k=1}^{n} a_{k j} D_{k}
$$

The symbol of $D_{j}$ as a first-order differential operator can be computed by the method given in $[18$, p. 63] as

$$
\sigma\left(D_{j}\right)\left(x^{0}, \xi^{0}\right)=-i D_{j}\left[\sum_{k \neq j}\left(x_{k} \xi_{k}^{0}-x_{k}^{0} \xi_{k}^{0}\right)\right]=-i \xi_{j}^{0}
$$

This is consistent with the definitions to be adopted below. 
Definition. The operators $R_{j}$ and $X_{j}$ are defined on $C^{\infty}\left(S^{n-1}\right)$ by

$$
\left(X_{j} f\right)(x)=x_{j} f(x), \quad\left(R_{j} f\right)(x)=\left(i D_{j} \Lambda^{-1} f\right)(x) .
$$

One can easily compute the transformation laws

$$
L_{a} X_{j} L_{a}^{-1}=\sum_{k} a_{k j} X_{k}, \quad L_{a} R_{j} L_{a}^{-1}=\sum_{k} a_{k j} R_{k} .
$$

We shall want to speak of the symbols of such operators as $C_{\infty}^{\infty}\left(S^{n-1}\right)$ singular operators, differential operators and powers of $\Lambda^{-1}$. It will be convenient to use the algebra $C Z\left(S^{n-1}\right)=\bigcup_{r} C Z_{r}\left(S^{n-1}\right)$ discussed in [19] and, except for certain changes in sign, the definition of $\sigma_{r}$ on $C Z_{r}\left(S^{n-1}\right) / C Z_{r-1}\left(S^{n-1}\right)$ given there. Briefly stated, an operator is in $C Z_{r}\left(S^{n-1}\right)$ if in each coordinate neighborhood $U$ the expression of $A$ in local coordinates gives rise to an operator $A_{U}=\sum_{j=0}^{\infty} A_{r-j}$ satisfying $\left(A_{r-j} f\right)^{\wedge}(\xi)=a_{r-j}(x, \xi) \hat{f}(\xi)$ for $f \in L^{2}\left(E_{n-1}\right)$ with support in the image $\Omega_{U}$ of $U$. We require that $a_{n-j}$ be $C^{\infty}$ for $\xi \neq 0$ and that $R_{k+1}=\sum_{j=0}^{k} A_{n-j}$ be bounded: $L_{s}^{2}\left(\Omega_{U}\right) \rightarrow L_{s+k+1}^{2}\left(\Omega_{U}\right)$. For $r<0$ let $\mathscr{T}_{r}$ denote the space of operators $K$ that (1) are given by kernels $k(x, z)$ which are $C^{\infty}$ for $z \neq 0$ and (2) are bounded: $L_{s}^{p} \rightarrow L_{s+r}^{p}$. By a modification of the proof of Lemma 4 in [21], we can extend the definition of $\sigma_{r}$ for $r \leqq 0$ to operators of the form $T+R$, where $T \in C Z_{r}\left(S^{n-1}\right)$ and $R \in \mathscr{T}_{r+1}\left(S^{n-1}\right)$, i.e., to $C Z_{r}\left(S^{n-1}\right)+\mathscr{T}_{r+1}\left(S^{n-1}\right)$. With this extension

$$
\sigma_{r}(A) \sigma_{s}(B)=\sigma_{r+s}(A B)
$$

continues to hold. Also, the function $\sigma_{0}$ on $C Z_{0}\left(S^{n-1}\right)+\mathscr{T}_{1}\left(S^{n-1}\right)$ agrees, except for sign, with the function $\sigma$ defined on $C_{\infty}^{\infty}\left(S^{n-1}\right)$ in [21]. In fact,

$$
\left[C Z_{0}\left(S^{n-1}\right)+\mathscr{T}_{1}\left(S^{n-1}\right)\right] / \mathscr{T}_{1}\left(S^{n-1}\right)=C_{\infty}^{\infty}\left(S^{n-1}\right),
$$

and both $\sigma_{0}$ and $\sigma$ vanish on $\mathscr{T}_{1}\left(S^{n-1}\right)$. Here we shall use the sign conventions arising from the formula for operators $H$ on $L^{p}\left(E_{n-1}\right)$

$$
\sigma(H)(x, \xi)=\lim _{\varepsilon \rightarrow 0} \int_{\varepsilon^{-1}>|z|>\varepsilon} k(x, z) e^{i z \xi} d z .
$$

(Here $k(x, z)$ is homogeneous of degree $-n+1$ in $z$ and has mean value 0 for each $x$.)

If $A$ is in $C Z_{r}\left(S^{n-1}\right)$, then $\sigma_{r}(A)$ satisfies the transformation law for a function defined on $T^{*}\left(S^{n-1}\right)$. More precisely, let $U$ be a coordinate neighborhood for $S^{n-1}$ and $\alpha: U \rightarrow \Omega_{U} \subseteq E_{n-1}$ a chart. If $\phi$ and $\psi$ are in $C^{\infty}\left(S^{n-1}\right)$, have their support in $U$, and are $\equiv 1$ on a neighborhood $V \subset U$, then there is an operator $H$ in $C Z_{r}\left(E_{n-1}\right) / C Z_{r-1}\left(E_{n-1}\right)$ supported in $\Omega_{U}$ (which we may assume to have compact closure in $E_{n-1}$ ) such that

$$
(\phi A \psi) f=H\left(f \circ \alpha^{-1}\right) \circ \alpha
$$

holds for functions $f$ supported in $U$. Moreover, for $x \in V$

$$
\sigma_{r}(A)\left(x, \xi_{x}\right)=\sigma_{r}(H)\left(\alpha(x),\left(d \alpha_{x}^{*}\right)^{-1} \xi_{x}\right),
$$


where $\xi_{x}$ is any element in the fibre over $x$, and $d \alpha_{x}^{*}$ is the adjoint of the differential of $\alpha$ at $x$. This formula is given on p. 264 of [19], where slightly different notation is employed.

Proposition 10. For $f \in C^{\infty}\left(S^{n-1}\right)$ and $a \in \mathrm{SO}(n) \operatorname{set}\left(L_{a} f\right)(x)=f\left(a^{-1} x\right), l_{a} x=a x$. Then if

$$
A \in C Z_{r}\left(S^{n-1}\right) / C Z_{r-1}\left(S^{n-1}\right), \quad \sigma\left(L_{a} A L_{a}^{-1}\right)(x, \xi)=\sigma_{r}(A)\left(a^{-1} x, a^{-1} \xi\right) .
$$

(Here we use (1.7) and the remarks following it.)

Proof. Let $U$ be a coordinate neighborhood for $S^{n-1}$ and let $\phi$ and $\psi$ be two $C^{\infty}\left(S^{n-1}\right)$ functions with support in $U$ that are $\equiv 1$ on the neighborhood $V$ of $x$. If $\alpha: U \rightarrow \Omega_{U}$ is a chart, then by (4.10)

$$
\sigma\left(L_{a} A L_{a}^{-1}\right)(x, \xi)=\sigma_{r}(H)\left(\alpha(x),\left(d \alpha_{x}^{*}\right)^{-1} \xi\right)
$$

where $H$ is an operator in $C Z_{r}\left(E_{n-1}\right)$ such that

$$
\left[\left(\phi L_{a} A L_{a}^{-1} \psi\right) f\right](x)=H\left(f \circ \alpha^{-1}\right) \circ \alpha .
$$

But for $x \in V$ and $f$ supported in $a^{-1} U$,

$$
\begin{aligned}
{\left[\left(L_{a}^{-1} \phi\right) A\left(L_{a}^{-1} \psi\right)\right] f\left(a^{-1} x\right) } & =\left[\left(\phi L_{a} A L_{a}^{-1} \psi\left(f \circ l_{a}^{-1}\right) \circ l_{a}\right]\left(a^{-1} x\right)\right. \\
& =\left[H\left(f \circ l_{a}^{-1} \circ \alpha^{-1}\right) \circ\left(\alpha \circ l_{a}\right)\right]\left(a^{-1} x\right),
\end{aligned}
$$

on applying (4.12) to $f \circ l_{a}$. Now (4.10) can be used with $\alpha$ replaced by the chart $\beta=\alpha \circ l_{a}$ for $a^{-1} U$ to obtain

$$
\begin{aligned}
\sigma_{r}(A)\left(a^{-1} x, a^{-1} \xi\right) & =\sigma_{r}(H)\left(\beta\left(a^{-1} x\right),\left(d \beta_{a}^{*}-1_{x}\right)^{-1} a^{-1} \xi\right) \\
& =\sigma_{r}(H)\left(\alpha(x),\left(d \alpha_{x}^{*}\right)^{-1}\left(d l_{a}^{*}\right)_{a}^{-1} a_{x} a^{-1} \xi\right) \\
& =\sigma_{r}\left(L A L_{a}^{-1}\right)\left(x, a a^{-1} \xi\right) .
\end{aligned}
$$

Proposition 10 is very useful when dealing with a system of operators transforming according to an irreducible representation $R$ of $\mathrm{SO}(n)$. For then if we know $\sigma(A)$ globally for one operator $A$ we can compute $\sigma\left(R_{a} A\right)$ globally for every $a \in \mathrm{SO}(n)$. Conversely, if for some open set $U$ of $S^{n-1}$ and each $A$ we know $\sigma(A)(x, \xi)$ for $x \in U$, then (4.11) can be used to compute $\sigma(A)$ globally for each $A$. In particular we may use the results of $\S$ III together with the fact that the system $\left\{c \Lambda^{-1} \mid c \in C\right\}$ transforms according to the trivial representation of $\mathrm{SO}(n)$ to compute $\sigma\left(\Lambda^{-1}\right)$.

Proposition 11. The operator $\Lambda^{-1}$ is in $\left[C Z_{-1}\left(S^{n-1}\right)+\mathscr{T}_{2}\left(S^{n-1}\right)\right] / \mathscr{T}_{2}\left(S^{n-1}\right)$. Its -1 order symbol is

$$
\sigma_{-1}\left(\Lambda^{-1}\right)(x, \xi)=|\xi|^{-1},
$$

where $(x, \xi) \in C S\left(S^{n-1}\right)$ is as in (1.5). 
Proof. It is enough to consider $\phi \Lambda^{-1} \psi$, where $\phi$ and $\psi$ are arbitrary $C^{\infty}$ functions that are $\equiv 1$ in $U_{\varepsilon}=\left\{x \in S^{n-1} \mid 1-\varepsilon<x_{n} \leqq 1\right\}$ and are supported in a slightly larger open set. By the remarks before (4.7) and Proposition 8,

$$
\sigma_{-1}\left(\Lambda^{-1}\right)\left(N, \xi_{N}\right)=\left(\xi_{1}^{2}+\xi_{2}^{2}+\cdots+\xi_{n-1}^{2}\right)^{-1 / 2}=\left|\xi_{N}\right|^{-1} .
$$

(See [21, p. 662] for $\sigma\left(\Lambda_{E}^{-1}\right)(\xi)$.) Now (4.15) follows from (4.11) together with the fact that if $a \xi_{N}=\left(\zeta_{1}, \ldots, \zeta_{n}\right)$, then $\left|a \xi_{N}\right|^{2}=\zeta_{1}^{2}+\cdots+\zeta_{n}^{2}=\xi_{1}^{2}+\cdots+\xi_{n-1}^{2}$. (Clearly, (4.11) continues to hold in the present situation.)

Since the symbol map (principal part) is an algebra homomorphism, it follows as a corollary of Proposition 11 that if $D$ is a differential operator of order $s$ on $S^{n-1}$ with $C^{\infty}$ coefficients, then

$$
\sigma_{0}\left(D \circ \Lambda^{-s}\right)(x, \xi)=\sigma_{s}(D)(x, \xi)|\xi|^{-s} .
$$

In particular, for the operators $R_{j}$ of (4.5)

$$
\sigma_{0}\left(R_{j}\right)(x, \xi)=\sigma_{0}\left(i D_{j} \circ \Lambda^{-1}\right)=\xi_{j}|\xi|^{-1} .
$$

Since the symbol of multiplication by a smooth function $a(x)$ is $a(x)$, we have

$$
\sigma_{0}\left(X_{j}\right)(x, \xi)=x_{j}
$$

Proposition 12. Let $q(x, \xi)$ be any polynomial such that for real numbers $t, u$ $q(t x, u \xi)=t^{r} u^{s} q(x, \xi)$. Then

$$
T_{q}=q(X, i D) \Lambda^{-s}
$$

is a $C_{\infty}^{\infty}\left(S^{n-1}\right)$ operator with

$$
\sigma\left(T_{q}\right)=q \mid C S\left(S^{n-1}\right) .
$$

Moreover, for $a \in \mathrm{SO}(n)$,

$$
L_{a} T_{q} L_{a}^{-1}=T_{q_{a}}
$$

Proof. The transformation law (4.21) for a polynomial $q=\sum_{|\alpha|=r ;|\beta|=s} c_{\alpha \beta} x^{\alpha} \xi^{\beta}$ follows from the transformation laws (4.6), which when rewritten become (4.21) for the polynomials $x$, and $\xi_{j}$. Also, (4.20) follows from (4.17) and (4.18), and the fact that $\sigma_{0}$ is a homomorphism.

Definition. If $q$ is an SO (n)-harmonic polynomial, then $T_{q}$ is the $C_{\infty}^{\infty}\left(S^{n-1}\right)$ operator defined by (4.19).

From now on we shall only speak of $T_{q}$ for SO (n)-harmonic polynomials $q$. In view of Proposition 12 we have achieved the construction $q \rightarrow T_{q}$ required for the definitions at the beginning of this section.

Before passing to the proof of Theorem 5 we establish some preliminary estimates.

LEMMA 9. Let $h \in C^{\infty}(\mathrm{SO}(n) / \mathrm{SO}(n-2))$ have the expansion $h=\sum_{m} h_{m}$, with $h_{m} \in \mathscr{H}_{m}$ (see (2.1)). Then as $|m|=m_{1}+\left|m_{2}\right| \rightarrow \infty$ 
(1) $\left\|h_{m}\right\|_{2}=O\left(|m|^{-k}\right)$ for any integer $k>0$;

(2) $d_{m}=$ degree $R^{m}=O\left(|m|^{2 n}\right)$.

Proof. For (1) we use the fact that $\mathscr{H}_{m}$ is the sum of eigenspaces of the (elliptic invariant Laplacian (or Casimir operator) $\Delta=\sum_{i<j} D_{i j}^{2}$ on $\mathrm{SO}(n)$. Since this operator is invariant under the right as well as the left action of SO $(n)$, the eigenvalue is the same for each summand in $\mathscr{H}_{m}$. This eigenvalue $\lambda(m)$ is of the form

$$
\lambda(m)=-\sum a_{i j} m_{i} m_{j}+\sum b_{k} m_{k},
$$

where $\left(a_{i j}\right)$ is a positive definite symmetric matrix (see [10, pp. 246-247]). From (4.22) and the projection formula

$$
\left(\pi_{m} h\right)(g)=d_{m} \int_{\text {so }(n)} h(a) \bar{\chi}_{m}\left(a^{-1} g\right) d a
$$

for $\pi_{m}$, the projection onto $\mathscr{H}_{m}$, we see that for any integer $k>0$

$$
\pi_{m}\left(\Delta^{k} h\right)=[\lambda(m)]^{k} \pi_{m} h=[\lambda(m)]^{k} h_{m} .
$$

(We used the facts that $\Delta$ is selfadjoint and that $\chi(a b)=\chi(b a)$.)

Hence for any integer $k>0$

$$
\sum_{m}[\lambda(m)]^{2 k}\left\|h_{m}\right\|_{2}^{2}<\infty,
$$

and the first part of the lemma follows.

The second assertion follows from an estimate using the degree formula for $R^{m}$ given in [1, p. 250]. (A trivial estimate gives $d_{m}=O\left(|m|^{n^{3}}\right)$, which is actually sufficient for our purposes.)

LEMMA 9. Let us choose a finite set of differential operators on $\operatorname{CS}\left(S^{n-1}\right)$ whose linear span is the space of all differential operators on $C S\left(S^{n-1}\right)$ of order $\leqq 2 k$. If $D$ is any element of this set and $h_{m} \in \mathscr{H}_{m}$, then

$$
\left|D h_{m}\right| \leqq C_{n, k}|m|^{2 n+2 k} \text {. }
$$

Proof. Any differential operator $D$ can be expressed in a small open set as the sum with smooth coefficients of iterates of differentiations given by the standard basis elements of the Lie algebra of $\mathrm{SO}(n)$. Since $C S\left(S^{n-1}\right)$ is compact, it is enough to prove an estimate like (4.24) for $D$ an iterate of order $\leqq 2 k$ of Lie algebra differentiations. Such operators $D$ commute with the Laplacian on SO $(n)$, and thus preserve the spaces $\mathscr{H}_{m}$. Write

$$
\left(D h_{m}\right)(g)=d_{m} \int_{\text {So }(n)}\left(D h_{m}\right)(a) \bar{\chi}_{m}\left(a^{-1} g\right) d a .
$$

Then for each $g \in \mathrm{SO}(n)$,

$$
\left|D h_{m}(g)\right| \leqq d_{m}\left\|D h_{m}\right\|_{2}\left\|\chi_{m}\right\|_{2} .
$$

But $\left\|\chi_{m}\right\|_{2}=1$ as a function of $a$ for each $g$, and the other factor can be majorized by $C\left\|(\Delta-1)^{k} h_{m}\right\|_{2}$, since $\Delta-1$ provides an isomorphism between $L_{r+2}^{p}(\mathrm{SO}(n))$ and 
$L_{r}^{p}(\mathrm{SO}(n))$ for $r \geqq 0$ (see [21, pp. 684-685]). In view of (4.22) and the argument following it, together with Lemma 8, we have (4.24).

Proof of Theorem 5. Using (2.6) and Theorem 2, let us write $\mathscr{H}_{m}$ as the (algebraic) direct sum of irreducible spaces:

$$
\mathscr{H}_{m}=\sum_{i=1}^{s(m)} H_{m, i}
$$

and choose an orthonormal basis $\left\{e_{i j}^{m}\right\}, j=1, \ldots, d_{m}$, for each $H_{m, i}$. Then if $T \in C_{\infty}^{\infty}\left(S^{n-1}\right)$,

$$
\sigma(T)=\sum_{m} \sum_{i=1}^{s(m)} \sum_{j=1}^{d_{m}} c_{i j}^{m} e_{i j}^{m}
$$

Let $\left\{\phi_{k}\right\}$ be any $C^{\infty}$ partition of unity for $S^{n-1}$. Let $T_{i j}^{m}$ denote the $C_{\infty}^{\infty}\left(S^{n-1}\right)$ operator constructed as in [21, p. 678] with the use of $\left\{\phi_{k}\right\}$ to have symbol $e_{i j}^{m}(x)$. Then

$$
\left\|T_{i j}^{m} f\right\|_{p} \leqq A B_{m}\|f\|_{p},
$$

where $A$ is a constant depending on $n, p$ and $\left\{\phi_{k}\right\}$, and $B_{m}$ is a bound for $e_{i j}^{m}(x)$ and its derivatives of order $\leqq 2 n-2$ with respect to cosphere variables. See [21, p. 678]. By (4.25), (4.26) and Lemma 9 the series $\sum_{m} \sum_{i, j} T_{i j}^{m}$ converges in $L^{p_{-}}$ operator norm.

Now let $T_{m, i}=\sum_{j=1}^{d_{m}} c_{i j}^{m} T_{i j}^{m}$. Because of the way we have defined $H_{m, i}$ there is a pair $(r, s)$ of indices such that $\sigma\left(T_{m, i}\right)=q_{m, i} \mid C S$, with $q_{m, i} \in P_{r, s}^{m}$. Setting $T_{m, i}^{\prime}=T_{q_{m, i}}$ for each $m, i$ we have

$$
\sigma(T)=\sigma\left(\sum_{m} \sum_{i} T_{m, i}\right), \quad \sigma\left(T_{m, i}^{\prime}\right)=\sigma\left(T_{m, i}\right),
$$

with $T_{m, i}^{\prime}$ lying in the canonical system $V_{r, s}^{m}$. The conclusion of Theorem 6 now follows from the fact that if $S$ is a $C_{\infty}^{\infty}\left(S^{n-1}\right)$ operator and $\sigma(S)=0$, then $S$ smooths.

Let $T$ be a bounded operator on a Banach space $B$ of measurable functions on $S^{n-1}$ whose norm $\|\cdot\|$ satisfies

(1) $\left\|f_{a}\right\|=\|f\|$;

(2) $a \rightarrow\left\|f_{a}\right\|$ is a continuous function of $a \in \mathrm{SO}(n)$ for each $f \in B$.

Then for each $a \in \mathrm{SO}(n), L_{a} T L_{a}^{-1}$ is bounded on $B$ with norm equal to that of $T$. Moreover, if $\phi$ is continuous on SO $(n)$, the integral $\int_{\mathrm{SO}(n)}\left(L_{a} T L_{a}^{-1}\right) f(x) \phi(a) d a$ defines a bounded operator on $B$. These remarks apply in particular to $B=L_{k}^{p}\left(S^{n-1}\right)$ for $1<p<\infty$ and $k \geqq 0$, and to any operator in $C Z\left(S^{n-1}\right)$.

Proof of Theorem 6. Let $V$ be any system of singular operators transforming according to the irreducible representation $R^{m_{0}}$ of $\mathrm{SO}(n)$. Fixing any element $T \in V$, we may write

$$
T=\sum_{m} \sum_{i}\left(T_{m, i}^{\prime}+S_{m, i}\right)
$$


as in Theorem 5. Since this series converges in $L^{2}$-operator norm, to compute

$$
d_{m_{0}} \int_{\mathrm{SO}(n)}\left(L_{a} T L_{a}^{-1}\right) f(x) \bar{\chi}_{m_{0}}(a) d a
$$

we may substitute (4.27) and integrate term by term. The resulting integrals involving $T_{m, i}^{\prime}$ will be zero if $m \neq m_{0}$, while the integral involving $S_{m, i}$ yields a smoothing operator whose norm does not exceed that of $T_{m, i}^{\prime}+S_{m, i}$. The first assertion can be justified by considering the integral

$$
\int_{\mathrm{SO}(n)} g(x) \int_{\mathrm{SO}(n)}\left(L_{a} T L_{a}^{-1} f\right)(x) \bar{\chi}_{m}(a) d a d x
$$

for $g \in C^{\infty}(\mathrm{SO}(n))$, and using Fubini's theorem and the Shur orthogonality relations. (See [1].) The second assertion follows from the $L^{p}$-operator convergence of $\sum T_{m_{0}, i}^{\prime}+S_{m, i}$, and the remarks made just before this proof.

Thus the right-hand side of (4.28) becomes $\sum_{i} T_{m_{0}, i}^{\prime}+S$, where $T_{m_{0}, i}^{\prime}$ lies in one of the canonical systems $V_{r, s}^{m}$ and $S$ smooths. By a similar argument the left-hand side of (4.28) is seen to be $T$. Thus $T=T_{q_{m_{0}}}+S$, with $q_{m_{0}} \in Q_{m_{0}}$. But for $n \geqq 4$ each $Q_{m}$ yields $m_{1}-\left|m_{2}\right|+1(2 m+1$ for $n=3)$ independent systems, and no more. This concludes the proof of Theorem 6 .

We remark that with the decomposition (2.6) of $Q_{m}$ each canonical system $V_{r, s}^{m}$ is described by the numbers $r$ and $s$ of multiplications and compensated differentiations.

\section{REFERENCES}

1. H. Boerner, Representations of groups, North-Holland, Amsterdam, 1963.

2. N. Bourbaki, Éléments de mathématique, Fasc. XXVII, Algèbre commutative, Hermann, Paris, 1961.

3. A. P. Calderón, Integrales singulares y sus aplicaciones a ecuaciones diferenciales hiperbolicas, Curos y Seminarios de Matemática, Fasc. 3, Universidad de Buenos Aires, Buenos Aires, 1960.

4. - Singular integrals, Bull. Amer. Math. Soc. 72 (1966), 427-465.

5. A. P. Calderón and A. Zygmund, Singular integral operators and differential equations, Amer. J. Math. 79 (1957), 901-921.

6. R. Godement, "Localites simples. I and II" in Séminaire H. Cartan et C. Chevalley, $8^{\ominus}$ année, École Norm. Sup., Paris, 1956.

7. S. Helgason, Differential geometry and symmetric spaces, Academic Press, New York, 1962.

8. - Some results on invariant theory, Bull. Amer. Math. Soc. 68 (1962), 367-371.

9. L. Hörmander, Pseudo-differential operators, Comm. Pure Appl. Math. 18 (1965), 501-517.

10. N. Jacobson, Lie algebras, Interscience, New York, 1966.

11. J. J. Kohn and L. Nirenberg, An algebra of pseudo-differential operators, Comm. Pure Appl. Math. 18 (1965), 269-305.

12. B. Kostant, Lie group representations on polynomial rings, Amer. J. Math. 85 (1963), 327-404.

13. S. Lang, Introduction to algebraic geometry, Interscience, New York, 1958. 
14. D. A. Levine, Singular integral operators on spheres, Ph.D. Thesis, Princeton University, Princeton, N. J., 1968.

15. S. G. Mihlin, Higher-dimensional singular integrals and integral equations, Fizmatgiz, Moscow, 1962; English transl., Pergamon Press, New York, 1965.

16. B. Muckenhoupt and E. M. Stein, Classical expansions and their relation to conjugate harmonic functions, Trans. Amer. Math. Soc. 118 (1965), 17-92.

17. F. D. Murnaghan, The theory of group representations, Johns Hopkins Press, Baltimore, Maryland, 1938.

18. R. S. Palais, "Differential operators on vector bundles" in Seminar on the Atiyah-Singer index theorem, Princeton Univ. Press, Princeton, N. J., 1965.

19. R. S. Palais and R. T. Seeley, "The construction of Int ${ }_{k}$ " in Seminar on the AtiyahSinger index theorem, Princeton Univ. Press, Princeton, N. J., 1965.

20. P. Samuel, Méthodes d'algèbre abstraite en géometrie algébrique, Ergebnisse der Mathematik und ihrer Grenzgebeite (N.F.), Heft 4, Springer-Verlag, Berlin, 1955.

21. R. T. Seeley, Singular integrals on compact manifolds, Amer. J. Math. 81 (1959), 658-690.

22. - _ - Spherical harmonics, Amer. Math. Monthly 73 (1966), no. 4, Part II, 115-121.

23. E. M. Stein, Integrales singulières et fonctions differentiables de plusieurs variables, Publications Mathématiques d'Orsay, 1967.

24. E. M. Stein and G. Weiss, Generalization of the Cauchy-Riemann equations and representations of the rotation group, Amer. J. Math. 90 (1968), 163-196.

25. H. Weyl, The classical groups, Princeton Univ. Press, Princeton, N. J., 1946.

26. _-_ Gruppentheorie und Quantenmechanik, 2nd ed., Hirzel, Leipzig, 1931; English transl., Dover, New York, 1950.

27. O. Zariski and P. Samuel, Commutative algebra, Vol. II, Van Nostrand, Princeton, N. J., 1960.

28. A. Zygmund, Trigonometric series, Cambridge Univ. Press, Cambridge, 1959.

UNIVERSITY OF MINNESOTA,

Minneapolis, Minnesota 